\title{
Identification of Railway Ballasted Track Systems from Dynamic Responses of In-service Trains
}

by

\author{
Zhu, X.Q. ${ }^{1}$, Law. S.S. ${ }^{2,3}$ and Huang, L. $^{4}$
}

\begin{abstract}
Railway track is one of the most important part of the railway system, and its condition monitoring is essential to ensure the safety of trains and reduce maintenance cost. An adaptive regularization approach is adopted in this paper to identify the parameters of the railway ballasted track system (substructure) from dynamic measurements on the in-service vehicles. The vehicle-track interaction system is modelled as a discrete spring-mass model on Winkler elastic foundation. Damage is defined as the stiffness reduction of the track due to foundation settlement, loosening in the rail fastener and lack of compaction of the ballast. Accelerometers are installed on the underframe of the train to capture the dynamic responses from which the interaction forces between the vehicle and the railway track are determined. The damage of the railway track can be detected via changes in the interaction force. Numerical results show that the proposed approach can identify all stiffness parameters successfully at a low moving speed and at a high sampling rate when measurement noise is involved.
\end{abstract}

Keywords: rail substructure; adaptive regularization; time domain; interaction force; moving sensor.

\footnotetext{
${ }^{1}$ Associate Professor, School of Civil and Environmental Engineering, University of Technology Sydney, Broadway, NSW 2007, Australia. Email: xinqun.zhu@uts.edu.au

2 Professor, School of Civil Engineering, Chongqing University, China. E-mail address: siu-seong.law@connect.polyu.hk

${ }^{3}$ Professor, Civil and Environment Engineering Department, Hong Kong Polytechnic University, Kowloon, Hunghum, Hong Kong, China. E-mail address: siu-seong.law@connect.polyu.hk

${ }^{4}$ Research Assistant, Civil and Environment Engineering Department, Hong Kong Polytechnic University, Kowloon, Hunghum, Hong Kong, China. E-mail address: 806045267@qq.com
} 


\section{$31 \quad$ Introduction}

Many countries have developed high-speed railroads to connect major cities especially in China. The unevenness of the railway track is important for the safety of the railway due to the high speed of the vehicle. Regular maintenance of the railway tracks has become a necessity, and monitoring on the conditions of the railway track network is conducted for early detection of damages. Some direct methods have been developed using impact hammer testing (Lam et al.,2012) and the Bayesian model updating has been used for the identification of the rail-sleeper-ballast system (Lam et al., 2017). This assessment of track is, however, expensive and difficult with the hammer impact test, and an efficient and economic inspection method is strongly in need.

Several research groups have studied the use of response from the passing vehicle instead of the response of the structure for the assessment of irregularities in the track. This indirect method is more convenient and cheaper than the direct method. Ishii et al. (2006) developed a low-cost railway monitoring system with the accelerometer installed directly on the floor of the train. The field measurements are capable of monitoring the railway track irregularities because the vehicle acceleration and track irregularity has a close correlation. Mizuno et al. (2008) used the same mobile sensing unit in an experiment, and the results indicated that the critical acceleration response on the floor of a passenger vehicle is a promising tool to capture the railway track disorders. Weston et al. (2007) installed the sensors on the bogie of an in-service vehicle to estimate the mean track alignment and lateral track irregularity. With and Bodare (2009) developed a rolling stiffness measurement vehicle to investigate the track condition and the point flexibility/stiffness of the track-embankment-subsoil system could be obtained in the frequency domain. More encouraging results can also be obtained by simultaneously measuring the force applied to an axle of the measuring vehicle and the resulting acceleration response. Cantero and Basu (2015) used the vertical accelerations of a moving train to detect local track irregularities. Isolated irregularities caused by infrastructural damage can be accurately identified with a wavelet-based automatic assessment methodology. Salva dor et al. (2016) located and distinguished some track vibration 
modes and singularities by the short Fourier transform of axle box accelerations. Lederman et al. (2017) presented a data-driven approach to monitor the track condition using the dynamic response of a passing train. Four features in the measured signal, i.e. the temporal frequency, spatial frequency, spatial domain and signal energy were used to detect the changes of the track. Oregui et al. (2017) monitored the bolt tightness conditions of rail joints by comparing the scalograms of measured axle box accelerations. Li et al. (2017) presented an overview of these signal-based track singularity monitoring techniques. It should be noted that all the above studies are not related to the assessment of the track and its substructure.

In the forward analysis of a train-track system, most researchers used the differential equation to derive the equation of motion of the rail substructures (Zhai and Cai, 1997; Uzzal et al., 2008; Mohammadzadeh et al., 2014 ) with the modal decomposition method. The number of mode shapes is required to be greater than or equal to 60 for good convergence results. All the initial and boundary conditions are assumed zero or stationary. These assumptions, however, result in incorrect solution in the first and last few seconds of the time history.

The railway substructures includes the rail fasteners, ballast and the foundation, and their condition assessment using the vehicle response will be studied. The rail is modeled as an infinitely long beam on discrete springs. Since the interaction forces exist at the wheel-rail contact points, the equations of motion of the vehicle and the track system are coupled, and it becomes possible to assess the conditions of the railway substructures by monitoring the vibration response of the vehicle. The vehicle moving on the rail track can be idealized as a series of lumped masses supported by the suspension systems (Yang and Yau, 1997). Only a half model of the vehicle is considered as the rail deformation and wheel-rail contact forces generated by the moving wheel in the two halves of the vehicle are very close to each other (Savini, 2010). The accelerometers located on the axle and body of the moving vehicle collect dynamic responses of the vehicle from which the interaction forces can be easily obtained. This time dependent interaction force is noted to be more sensitive to local system changes than other responses (Law et al., 2010). 
This paper addresses the more practical problem of condition assessment of the track and its substructure whereby the unknown parameters to be identified are plenty and their damage effects are coupling with each other. The loosening of rail fasteners, the lack of compaction of ballast and settlement of the foundation are all modelled as stiffness change in a component of the track substructure. The Winkler elastic foundation (Vale and Calcada, 2013) is used to model the track foundation. The model on the track and its substructure is used for the first time in the system identification of the track system. Adaptive Tikhonov regularization (Li and Law, 2010) is adopted in the solution of the inverse identification problem. Numerical examples show that all these stiffness parameters can be identified satisfactory with a slow moving vehicle and a higher sampling rate with or without noise in the measurement.

\section{Vehicle and track interaction model}

\section{Vehicle model}

A train vehicle and rail track interactive system is shown in Figure 1 (Zhai and Cai, 1997). The train travels over the track with a constant speed $v$. It is modeled as a series of sprung masses supported by the suspension systems (Yang and Yau, 1997). The train vehicle consist of one-axle trailer with the bogie mass $m_{v 1}$ and wheel mass $m_{v 2}$ connected to the suspension damper $c_{v}$ and a suspension spring $k_{v}$. The rolling and pitching motions of the vehicle are ignored in this quarter vehicle model. The equation of motion of the vehicle can thus be written as

$$
\begin{gathered}
m_{v 1} \ddot{y}_{v 1}(t)+c_{v}\left(\dot{y}_{v 1}(t)-\dot{y}_{v 2}(t)\right)+k_{v}\left(y_{v 1}(t)-y_{v 2}(t)\right)=0 \\
m_{v 2} \ddot{y}_{v 2}(t)+c_{v}\left(\dot{y}_{v 2}(t)-\dot{y}_{v 1}(t)\right)+k_{v}\left(y_{v 2}(t)-y_{v 1}(t)\right)+P_{c}(t)-F_{v}=0
\end{gathered}
$$

where $\boldsymbol{y}_{v 1}(t)$ and $\boldsymbol{y}_{v 2}(t)$ are the motion of vehicle bogie and wheel, respectively. $P_{c}(t)$ is the wheel-rail contact force. $\mathrm{F}_{v}=\left(m_{v 1}+m_{v 2}\right) g$ is the mass of the train, $g$ is the acceleration of gravity. It is assumed that the wheel and rail contact point lies on the vertical centerline of the wheel. Substituting Eq. (1) into Eq. (2), the contact force can be written as

$$
P_{c}(t)=F_{v}-m_{v 1} \ddot{y}_{v 1}(t)-m_{v 2} \ddot{y}_{v 2}(t)
$$



vertical acceleration responses.

$$
\left[\begin{array}{cc}
m_{v 1} & 0 \\
0 & m_{v 2}
\end{array}\right]\left\{\begin{array}{c}
\ddot{y}_{v 1}(t) \\
\ddot{y}_{v 2}(t)
\end{array}\right\}+\left[\begin{array}{cc}
c_{v} & -c_{v} \\
-c_{v} & c_{v}
\end{array}\right]\left\{\begin{array}{l}
\dot{y}_{v 1}(t) \\
\dot{y}_{v 2}(t)
\end{array}\right\}+\left[\begin{array}{cc}
k_{v} & -k_{v} \\
-k_{v} & k_{v}
\end{array}\right]\left\{\begin{array}{l}
y_{v 1}(t) \\
y_{v 2}(t)
\end{array}\right\}=\left\{\begin{array}{c}
0 \\
-P_{c}(t)+F_{v}
\end{array}\right\}
$$

112

Let $\quad \mathbf{M}_{v}=\left[\begin{array}{cc}m_{v 1} & 0 \\ 0 & m_{v 2}\end{array}\right], \quad \mathbf{C}_{v}=\left[\begin{array}{cc}c_{v} & -c_{v} \\ -c_{v} & c_{v}\end{array}\right], \quad \mathbf{K}_{v}=\left[\begin{array}{cc}k_{v} & -k_{v} \\ -k_{v} & k_{v}\end{array}\right], \quad \mathbf{y}_{v}(t)=\left\{\begin{array}{l}y_{v 1}(t) \\ y_{v 2}(t)\end{array}\right\}$,

$P_{v}(t)=-P_{c}(t)+F_{v}$. Then, Eq. (4) can be rewritten as

$$
\mathbf{M}_{v} \ddot{\mathbf{y}}_{v}(t)+\mathbf{C}_{v} \dot{\mathbf{y}}_{v}(t)+\mathbf{K}_{v} \mathbf{y}_{v}(t)=\mathbf{D} P_{v}(t)
$$

115

where $\mathbf{D}=\left[\begin{array}{ll}0 & 1\end{array}\right]^{T}$ is the mapping vector.

The contact force between the wheel and the track is modeled with the linear Hertzian model which consists of the wheel and rail contact through a single linear spring. It can be expressed as (Vale and Calcada, 2013)

$$
P_{c}(t)=\left\{\begin{array}{c}
K_{H} \delta Z(t), \quad \delta Z(t) \geq 0 \\
0, \quad \delta Z(t)<0
\end{array}\right.
$$

where $\mathrm{K}_{\mathrm{H}}$ is the wheel and rail contact coefficient. $\delta Z(t)$ is the elastic wheel deformation in the vertical direction as

$$
\delta Z(t)=y_{v 2}(t)-y_{r}(x, t)-r(t)
$$

where $y_{r}(x, t)$ is the vertical rail deflections. $r(t)$ is the irregularities component of the wheel and rail contact surface. Many types of geometric irregularities can be adopted. This paper considers the effect of two most influential factors, i.e. the wheel flat and the rail corrugation. The wheel flat which enters the contact area between the wheel and the rail can be expressed as a cosine function (Wu and Thompson, 2002) as

$$
r(t)=\frac{1}{2} D_{f}\left[1-\cos \left(\frac{2 \pi z(t)}{L_{f}}\right)\right]
$$


contact point on the rail. If the train speed is high, loss of contact may occur with the existence of wheel flat.

A sine function is used to represent a rail corrugation as (Savini, 2010)

$$
r(t)=A \sin \left(\frac{2 \pi x(t)}{L}\right)
$$

where $A$ is the irregularity amplitude and $L$ is the total length travelled by the vehicle in the analysis.

\section{The Track Model}

The rail in the track is modeled as an infinitely long beam on a series of discrete springs, dampers and masses. The rail is discretely supported on the track substructure consisting of the sleepers, ballast and the foundation elements as shown in Figure 1 (Zhai and Cai, 1997; Uzzal et al., 2008) .

These three components form one unit of track substructure which connects to adjacent unit via the shear spring in the ballast layer. The rail beam is modeled as a free Euler-Bernoulli beam. Equation of motion of the rail can be written as

$$
\mathbf{M}_{r} \ddot{\mathbf{y}}_{r}(t)+\mathbf{C}_{r} \dot{\mathbf{y}}_{r}(t)+\mathbf{K}_{r} \mathbf{y}_{r}(t)=\mathbf{R}^{T}(t) P_{c}(t)-\mathbf{F}_{r}(t)
$$

where $\mathbf{M}_{r} \quad \mathbf{C}_{r}$ and $\mathbf{K}_{r}$ are the mass, damping and stiffness matrices of the rail respectively. The Rayleigh damping model (Bathe, 1982) $\mathbf{C}_{r}=a_{1} \mathbf{M}_{r}+a_{2} \mathbf{K}_{r}$ is adopt for the rail, where $a_{1}$ and $a_{2}$ are the two Rayleigh damping coefficients. $\boldsymbol{y}_{r}(t), \dot{\boldsymbol{y}}_{r}(t)$ and $\ddot{\boldsymbol{y}}_{r}(t)$ are rail displacement, velocity and acceleration responses, respectively. $\mathbf{R}(t)=\left\{0,0, \cdots, \mathbf{R}_{i}(t), \cdots, 0\right\}$ is the time-varying vector. Vector $\mathbf{R}_{i}(t)$ is the shape functions in the $i$ th element of the rail where the moving vehicle is located at time instant $t$, and it can be expressed as $\mathbf{R}_{i}(t)=\left\{1-3 \xi^{2}+2 \xi,\left(\xi-2 \xi^{2}+\xi^{3}\right) l_{e}, 3 \xi^{2}-2 \xi^{3},\left(-\xi^{2}+\xi^{3}\right) l_{e}\right\}^{T}$, with $\xi=\left(x(t)-x_{i}\right) / l_{e}, x_{i}=(i-1) l_{e}$ and $l_{e}$ is the length of the element. The rail and sleepers interface force vector is $\mathbf{F}_{r}(t)=\sum_{i=1}^{N} \mathrm{~F}_{r i}(t) \delta\left(x-x_{i}\right)$, where $N$ is the number of sleeper underneath the rail, $\delta(x)$ is the Dirac 
153

154

155

156

157

158

159

160

161

162 where

$$
\left[\begin{array}{ll}
\mathbf{M}_{r} & 0
\end{array}\right]\left\{\begin{array}{l}
\ddot{\mathbf{y}}_{r}(t) \\
\ddot{\mathbf{y}}_{s}(t)
\end{array}\right\}+\left[\begin{array}{ll}
\mathbf{C}_{r}+\mathbf{C}_{p r} & -\mathbf{C}_{p s}
\end{array}\right]\left\{\begin{array}{l}
\dot{\mathbf{y}}_{r}(t) \\
\dot{\mathbf{y}}_{s}(t)
\end{array}\right\}+\left[\begin{array}{l}
\mathbf{K}_{r}+\mathbf{K}_{p r}+\mathbf{R}^{T}(t) \mathbf{R}(t) \mathbf{K}_{\mathrm{H}}-\mathbf{K}_{p s}
\end{array}\right]\left\{\begin{array}{l}
\mathbf{y}_{r}(t) \\
\mathbf{y}_{s}(t)
\end{array}\right\}=\mathbf{R}^{T}(t) \mathbf{K}_{\mathrm{H}}\left(y_{v 2}(t)-r(t)\right)
$$

delta function, $x_{i}$ is the horizontal coordinate of the $i$ th sleeper from the left end. $\mathrm{F}_{r i}(t)$ is the $i$ th interface force between the rail and the sleeper as

$$
\mathrm{F}_{r i}(t)=k_{p i}\left(y_{r}\left(x_{i}, t\right)-y_{s i}(t)\right)+c_{p i}\left(\dot{y}_{r}\left(x_{i}, t\right)-\dot{y}_{s i}(t)\right)
$$

where $k_{p i}$ and $c_{p i}$ are stiffness and damping of the $i$ th rail fastener respectively. $y_{s i}(t)$ and $\dot{y}_{s i}(t)$ are respectively the displacement and velocity responses of the $i$ th sleeper in the vertical direction at time instant $t$.

Substituting Eqs. (6), (7) and (11) into Eq.(10), we have

$$
\begin{aligned}
& \text { where } \\
& \quad \mathbf{M}_{r}=\left[\begin{array}{cccc}
m_{r 1} & 0 & \cdots & 0 \\
0 & m_{r 2} & \cdots & 0 \\
\vdots & \vdots & \ddots & \vdots \\
0 & 0 & \cdots & m_{r N}
\end{array}\right], \mathbf{C}_{p r}=\left[\begin{array}{cccccccc}
c_{p 1} & 0 & 0 & 0 & \cdots & 0 & 0 \\
0 & 0 & 0 & 0 & \cdots & 0 & 0 \\
0 & 0 & c_{p 2} & 0 & \cdots & 0 & 0 \\
0 & 0 & 0 & 0 & \cdots & 0 & 0 \\
\vdots & \vdots & \vdots & \vdots & \ddots & \vdots & \vdots \\
0 & 0 & 0 & 0 & 0 & c_{p N} & 0 \\
0 & 0 & 0 & 0 & 0 & 0 & 0
\end{array}\right], \\
& \mathbf{K}_{p r}=\left[\begin{array}{ccccccc}
k_{p 1} & 0 & 0 & 0 & \cdots & 0 & 0 \\
0 & 0 & 0 & 0 & \cdots & 0 & 0 \\
0 & 0 & k_{p 2} & 0 & \cdots & 0 & 0 \\
0 & 0 & 0 & 0 & \cdots & 0 & 0 \\
\vdots & \vdots & \vdots & \vdots & \ddots & \vdots & \vdots \\
0 & 0 & 0 & 0 & 0 & k_{p N} & 0 \\
0 & 0 & 0 & 0 & 0 & 0 & 0
\end{array}\right], \mathbf{C}_{p s}=\left[\begin{array}{cccccc}
c_{p 1} & 0 & \cdots & 0 \\
0 & 0 & \cdots & 0 \\
0 & c_{p 2} & \cdots & 0 \\
0 & 0 & \cdots & 0 \\
0 & 0 & \cdots & 0 \\
\vdots & \vdots & \ddots & \vdots \\
0 & 0 & 0 & c_{p N}
\end{array}\right], \mathbf{K}_{p s}=\left[\begin{array}{ccccc}
k_{p 1} & 0 & \cdots & 0 \\
0 & 0 & \cdots & 0 \\
0 & k_{p 2} & \cdots & 0 \\
0 & 0 & \cdots & 0 \\
0 & 0 & \cdots & 0 \\
\vdots & \vdots & \ddots & \vdots \\
0 & 0 & 0 & k_{p N}
\end{array}\right]
\end{aligned}
$$

164 165 166 where $m_{r i}$ is the rail mass in the $i$ th element.

Equation of motion of the sleepers can be written as

$$
\begin{array}{r}
m_{s i} \ddot{y}_{s i}(t)+\left(c_{p i}+c_{b i}\right) \dot{y}_{s i}(t)+\left(k_{p i}+k_{b i}\right) y_{s i}(t)-c_{b i} \dot{y}_{b i}(t)-k_{b i} y_{b i}(t)-c_{p i} \dot{y}_{r}\left(x_{i}, t\right)-k_{p i} y_{r}\left(x_{i}, t\right)=0 \\
(i=1,2, \cdots, N)
\end{array}
$$

167 where $k_{b i}$ and $c_{b i}$ are the $i$ th ballast stiffness and damping, respectively. For the entire length of 168 the system model, Eq. (12) can be written as 


$$
\left[\begin{array}{lll}
0 & \mathbf{M}_{s} & 0
\end{array}\right]\left\{\begin{array}{l}
\ddot{\mathbf{y}}_{r}(t) \\
\ddot{\mathbf{y}}_{s}(t) \\
\ddot{\mathbf{y}}_{b}(t)
\end{array}\right\}+\left[\begin{array}{lll}
-\mathbf{C}_{p s}^{T} & \mathbf{C}_{p b} & -\mathbf{C}_{b b}
\end{array}\right]\left\{\begin{array}{l}
\dot{\mathbf{y}}_{r}(t) \\
\dot{\mathbf{y}}_{s}(t) \\
\dot{\mathbf{y}}_{b}(t)
\end{array}\right\}+\left[\begin{array}{lll}
-\mathbf{K}_{p s}^{T} & \mathbf{K}_{p b} & -\mathbf{K}_{b b}
\end{array}\right]\left\{\begin{array}{l}
\mathbf{y}_{r}(t) \\
\mathbf{y}_{s}(t) \\
\mathbf{y}_{b}(t)
\end{array}\right\}=0
$$

171 where

172

$$
\begin{gathered}
\mathbf{M}_{s}=\left[\begin{array}{cccc}
m_{s 1} & 0 & \cdots & 0 \\
0 & m_{s 2} & \cdots & 0 \\
\vdots & \vdots & \ddots & \vdots \\
0 & 0 & \cdots & m_{s N}
\end{array}\right], \mathbf{C}_{p b}=\left[\begin{array}{ccccc}
c_{p 1}+c_{b 1} & 0 & \cdots & 0 \\
0 & c_{p 2}+c_{b 2} & \cdots & 0 \\
\vdots & \vdots & \ddots & \vdots \\
0 & 0 & \cdots & c_{p N}+c_{b N}
\end{array}\right], \\
\mathbf{K}_{p b}=\left[\begin{array}{cccccc}
k_{p 1}+k_{b 1} & 0 & \cdots & 0 \\
0 & k_{p 2}+k_{b 2} & \cdots & 0 \\
\vdots & \vdots & \ddots & \vdots \\
0 & & 0 & \cdots & k_{p N}+k_{b N}
\end{array}\right], \mathbf{C}_{b b}=\left[\begin{array}{cccc}
c_{b 1} & 0 & \cdots & 0 \\
0 & c_{b 2} & \cdots & 0 \\
\vdots & \vdots & \ddots & \vdots \\
0 & 0 & \cdots & c_{b N}
\end{array}\right], \\
\mathbf{K}_{b b}=\left[\begin{array}{cccc}
k_{b 1} & 0 & \cdots & 0 \\
0 & k_{b 2} & \cdots & 0 \\
\vdots & \vdots & \ddots & \vdots \\
0 & 0 & \cdots & k_{b N}
\end{array}\right]
\end{gathered}
$$

174 where $m_{s i}$ is the mass of the $i$ th sleeper. $\boldsymbol{y}_{b}(t) 、 \dot{\boldsymbol{y}}_{b}(t)$ and $\ddot{\boldsymbol{y}}_{b}(t)$ are the displacement, velocity 175 and acceleration responses, respectively of the ballast.

$176 \quad$ Equation of motion of the ballasts can be written as

$$
\begin{array}{lr}
m_{b i} \ddot{y}_{b i}(t)+\left(c_{b i}+c_{f i}+c_{w i}+c_{w(i+1)}\right) \dot{y}_{b i}(t)+\left(k_{b i}+k_{f i}+k_{w i}+k_{w(i+1)}\right) y_{b i}(t)-c_{b i} \dot{y}_{s i}(t)-k_{b i} y_{s i}(t)- \\
c_{w i} \dot{y}_{b(i+1)}(t)-k_{w i} y_{b(i+1)}(t)-c_{w i} \dot{y}_{b(i-1)}(t)-k_{w i} y_{b(i-1)}(t)=0 & (i=1,2, \cdots, N)
\end{array}
$$

178

179

For the entire length of the system model, Eq .(13) can be written as

180

$$
\left[\begin{array}{ll}
0 & \mathbf{M}_{b}
\end{array}\right]\left\{\begin{array}{c}
\ddot{\mathbf{y}}_{s}(t) \\
\ddot{\mathbf{y}}_{b}(t)
\end{array}\right\}+\left[\begin{array}{ll}
-\mathbf{C}_{b b} & \mathbf{C}_{f w}-\mathbf{C}_{w b}
\end{array}\right]\left\{\begin{array}{c}
\dot{\mathbf{y}}_{s}(t) \\
\dot{\mathbf{y}}_{b}(t)
\end{array}\right\}+\left[\begin{array}{ll}
-\mathbf{K}_{b b} & \mathbf{K}_{f w}-\mathbf{K}_{w b}
\end{array}\right]\left\{\begin{array}{c}
\mathbf{y}_{s}(t) \\
\mathbf{y}_{b}(t)
\end{array}\right\}=0
$$

181 where $\mathbf{M}_{b}=\left[\begin{array}{cccc}m_{b 1} & 0 & \cdots & 0 \\ 0 & m_{b 2} & \cdots & 0 \\ \vdots & \vdots & \ddots & \vdots \\ 0 & 0 & \cdots & m_{b N}\end{array}\right]$, 


$$
\begin{aligned}
& \mathbf{C}_{f w}=\left[\begin{array}{cccccc}
c_{b 1}+c_{f 1}+c_{w 1}+c_{w 2} & -c_{w 1} & 0 & \cdots & 0 & 0 \\
-c_{w 2} & c_{b 2}+c_{f 2}+c_{w 2}+c_{w 3} & -c_{w 2} & \cdots & 0 & 0 \\
\vdots & \vdots & \vdots & \ddots & 0 & 0 \\
0 & 0 & 0 & \cdots & -c_{w N} & c_{b N}+c_{f N}+c_{w N}+c_{w(N+1)}
\end{array}\right] \\
& \mathbf{K}_{f w}=\left[\begin{array}{cccccc}
k_{b 1}+k_{f 1}+k_{w 1}+k_{w 2} & -k_{w 1} & 0 & \cdots & 0 & 0 \\
-k_{w 2} & k_{b 2}+k_{f 2}+k_{w 2}+k_{w 3} & -k_{w 2} & \cdots & 0 & 0 \\
\vdots & \vdots & \vdots & \ddots & 0 & 0 \\
0 & 0 & 0 & \cdots & -k_{w N} & k_{b N}+k_{f N}+k_{w N}+k_{w(N+1)}
\end{array}\right] .
\end{aligned}
$$

184 where $m_{b i}$ is the mass of the $i$ th ballast. $k_{w i}$ and $c_{w i}$ are the $i$ th ballast shearing stiffness and 185 damping, respectively. $k_{f i}$ and $c_{f i}$ are the stiffness and damping, respectively of the $i$ th 186 foundation component. in the following matrix form as

$$
\mathbf{M}_{t r} \ddot{\mathbf{Y}}_{t r}(t)+\mathbf{C}_{t r} \dot{\mathbf{Y}}_{t r}(t)+\mathbf{K}_{t r} \mathbf{Y}_{t r}(t)=\mathbf{L}^{T}(t) \mathrm{K}_{\mathrm{H}}\left(y_{v 2}(t)-r(t)\right)
$$

$190 \quad$ where $\quad \mathbf{Y}_{t r}=\left[\begin{array}{c}\boldsymbol{y}_{r}(t) \\ \boldsymbol{y}_{s}(t) \\ \boldsymbol{y}_{b}(t)\end{array}\right], \quad \boldsymbol{y}_{s}(t)=\left[\begin{array}{c}y_{s 1}(t) \\ \vdots \\ y_{s N}(t)\end{array}\right], \quad \boldsymbol{y}_{b}(t)=\left[\begin{array}{c}y_{b 1}(t) \\ \vdots \\ y_{b N}(t)\end{array}\right]$, and $\mathbf{L}(t)=[\mathbf{R}(t), 0, \cdots, 0]$ is a $2 N \times 1$

195 where $\mathbf{M}=\left[\begin{array}{cc}\mathbf{M}_{t r} & 0 \\ 0 & \mathbf{M}_{v}\end{array}\right], \quad \mathbf{C}=\left[\begin{array}{cc}\mathbf{C}_{t r} & 0 \\ 0 & \mathbf{C}_{v}\end{array}\right]$ and $\mathbf{K}=\left[\begin{array}{cc}\mathbf{K}_{t r} & \mathbf{K}_{t r-v} \\ \mathbf{K}_{v-t r} & \mathbf{K}_{v v}\end{array}\right]$ are the generalized mass, 196 damping and stiffness matrices of the system, respectively. $\mathbf{K}_{t r-v}=\left[\begin{array}{ll}0 & -\mathbf{L}^{T}(t) \mathbf{K}_{\mathrm{H}}\end{array}\right]$ and 197 $\mathbf{K}_{v-t r}=\left[\begin{array}{c}0 \\ -\mathbf{L}(t) \mathrm{K}_{\mathrm{H}}\end{array}\right], \quad \mathbf{K}_{v v}=\left[\begin{array}{cc}k_{v} & -k_{v} \\ -k_{v} & k_{v}+\mathrm{K}_{\mathrm{H}}\end{array}\right]$. The term $\boldsymbol{Q}(t)=\left[\begin{array}{llll}\mathbf{L}^{T}(t) \mathrm{K}_{\mathrm{H}} r(t) & 0 & \mathrm{~K}_{\mathrm{H}} r(t)+\mathrm{F}_{v}\end{array}\right]^{T}$ is 198 the corresponding force vector containing components of the wheel-rail contact force and the

mapping vector.

Combining Eqs. (5), (6) and (14), the coupled equation of the motion of the vehicle-track system can be obtained as (Henchi et al., 1988)

$$
\mathbf{M} \ddot{\mathbf{Y}}(t)+\mathbf{C} \dot{\mathbf{Y}}(t)+\mathbf{K Y}(t)=\boldsymbol{Q}(t)
$$
external force. The dynamic response of Eq. (15) can be calculated from the explicit Newmark- $\beta$ 
200

time-stepping integration method (Liu et al., 2014).

201 et al., 2011) linear model can be written as

For an unstable track foundation, the stiffness can be written as Eqs. (16b) and (16c) (Kacar

$$
k_{f}(x)=k_{f 0}
$$

for a linear and parabolic distributions and $x$ is the coordinate along the rail direction. The constant $\alpha \in[0,1]$ denotes the magnitude of settlement due to the extra flexibility. Assuming that there is only one zone of foundation settlement along the track, the mid-length of the reduced stiffness distribution is $x_{d}$, and the length of the settlement zone is $w_{d}$. The stiffness of foundation at the middle of the zone is therefore $k_{f 0}(1-\alpha)$. The stiffness distribution of the foundation with this

$$
k_{f}\left(x, x_{d}, w_{d}\right)=\left\{\begin{array}{cc}
k_{f 0}, & 0 \leq x \leq x_{d}-w_{d} / 2 \\
k_{f 0}\left(1-2 \alpha\left(x-x_{d}+w_{d} / 2\right) / w_{d}\right), & x_{d}-w_{d} / 2 \leq x \leq x_{d} \\
k_{f 0}\left(1+2 \alpha\left(x-x_{d}-w_{d} / 2\right) / w_{d}\right), & x_{d} \leq x \leq x_{d}+w_{d} / 2 \\
k_{f 0}, & x_{d}+w_{d} / 2 \leq x \leq L
\end{array}\right.
$$

and that for the foundation with a parabolic distribution model can be written as 


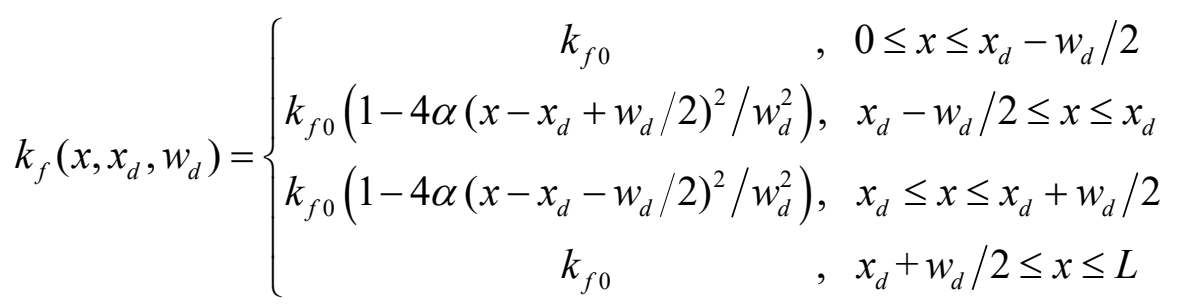

where $L$ is the total length of the foundation considered in the analysis. If there are $m$ multiple zones of settlement, the central location of the settlement zones becomes $x_{d}=\left[x_{d 1}, x_{d 2}, \cdots, x_{d m}\right]$ with the length of zones $w_{d}=\left[w_{d 1}, w_{d 2}, \cdots, w_{d m}\right]$. The stiffness for the $i$ th spring can be obtained from Eqs. (16a) to (17b) as

$$
k_{f i}=\int_{(i-1) \times l_{f}}^{i \times l_{f}} k_{f}\left(x, x_{d}, w_{d}\right) d x
$$

where $l_{f}$ is the length of finite element and it equals to $l_{e}$ for the rail in the present study.

In the present inverse analysis, the foundation settlement can be modeled as due to local flexibility, and the foundation stiffness identification can be interpreted as the identification of a stiffness change as

$$
k_{f i}=\left(1-\zeta_{f i}\right) k_{f}, \quad(i=1,2, \cdots, N)
$$

where $k_{f}$ is the $i$ th spring stiffness of the foundation without settlement. $\zeta_{f i}$ represents the stiffness reduction of the $i$ th spring stiffness. $\zeta_{f i} \leq 0.0$ indicates the undamaged condition and $\zeta_{f i} \geq 1.0$ indicates a total loss of the spring stiffness.

\section{Model of other system components}

For the connection between the rail and sleepers, such as the rail fastener, the local damage can be modeled as a spring stiffness reduction as

$$
k_{p i}=\left(1-\zeta_{p i}\right) k_{p i}^{0}, \quad(i=1,2, \cdots, N)
$$

where $k_{p i}^{0}$ is the $i$ th spring stiffness in the intact state. $\zeta_{p i}$ represents the fraction of stiffness reduction.

Similar model can be used to denote the lack of compaction in the ballast associating with a 
local flexibility in the media, and it can be expressed as

$$
k_{b i}=\left(1-\zeta_{b i}\right) k_{b i}^{0}, \quad(i=1,2, \cdots, N)
$$

where $k_{b i}^{0}$ is the $i$ th spring stiffness in the intact state and $\zeta_{b i}$ represents the fraction of stiffness reduction.

The shearing stiffness and damping of ballast are assumed less significant in contributing to the vertical deformation of the substructure and are thus ignored (Lam et al., 2012) in this study.

\section{Identification algorithm}

A change in the track substructure can be modelled by a vector of stiffness parameters $\zeta=\left[\zeta_{p 1}, \zeta_{p 2}, \cdots, \zeta_{p n}, \zeta_{b 1}, \zeta_{b 2}, \cdots, \zeta_{b n}, \zeta_{f 1}, \zeta_{f 2}, \cdots, \zeta_{f n}\right]$, and thus the system identification can be treated as an optimization problem. The contact force between the track and the vehicle, $\mathbf{P}_{c}^{\text {meas }}$ can be obtained from the measured acceleration response of the vehicle. The contact force without settlement, $\mathbf{P}_{c}^{c a l}(\zeta)$, can be calculated from the theoretical acceleration responses with an estimated vector of stiffness parameters $\zeta$. The damage identification equation for the $(j+1)$ th iteration can be defined as

$$
\mathbf{S}^{j} \boldsymbol{\varsigma}^{j+1}=\mathbf{P}_{c}^{\text {meas }}-\mathbf{P}_{c}^{c a l}\left(\boldsymbol{\varsigma}^{j}\right)=\Delta \mathbf{P}_{c}^{j}
$$

where $\boldsymbol{\zeta}^{j}$ is the identified stiffness parameter vector in the $j$ th iteration. $\boldsymbol{S}^{j}$ is the corresponding sensitivity matrix $\left.\frac{\partial \mathbf{P}_{c}^{c a l}(\varsigma)}{\partial \varsigma}\right|_{\xi=\varsigma^{j}}$. The first partial derivative of the contact force with respect to the stiffness parameter vector can be obtained by taking the first derivative with respect to the parameter vector on both sides of Eq. (3) as

$$
\frac{\partial \mathbf{P}_{c}^{c a l}(t)}{\partial \boldsymbol{\zeta}}=-m_{v 1} \frac{\partial \ddot{y}_{v 1}(t)}{\partial \zeta}-m_{v 2} \frac{\partial \ddot{y}_{v 2}(t)}{\partial \zeta}
$$

where the reference to the stiffness parameter in the functions have been removed, and $\frac{\partial \ddot{y}_{v 1}(t)}{\partial \zeta}$ and 
$261 \frac{\partial \ddot{y}_{v 2}(t)}{\partial \zeta}$ can be obtained by taking the first derivative with respect to the parameter vector on both

262 sides of the coupled equation in Eq. (15) as

$$
\mathbf{M} \frac{\partial \ddot{\mathbf{Y}}(t)}{\partial \boldsymbol{\zeta}}+\mathbf{C} \frac{\partial \dot{\mathbf{Y}}(t)}{\partial \boldsymbol{\zeta}}+\mathbf{K} \frac{\partial \mathbf{Y}(t)}{\partial \boldsymbol{\zeta}}=-\frac{\partial \mathbf{K}}{\partial \boldsymbol{\zeta}} \mathbf{Y}(t)
$$

264 where $\frac{\partial \ddot{\mathbf{Y}}(t)}{\partial \zeta}$ contains the terms $\frac{\partial \ddot{y}_{v 1}(t)}{\partial \zeta}$ and $\frac{\partial \ddot{y}_{v 2}(t)}{\partial \zeta}$, and $\frac{\partial \mathbf{K}}{\partial \zeta}$. Then the response sensitivities $265 \frac{\partial \ddot{\mathbf{Y}}(t)}{\partial \zeta}$ can also be calculated from the Newmark integration method.

the following cost function as

$$
J\left(\zeta^{j+1}\right)=\left\|\boldsymbol{S}^{j} \zeta^{j+1}-\Delta \mathbf{P}_{c}^{j}\right\|_{2}^{2}
$$

In the adaptive Tikhonov regularization ( $\mathrm{Li}$ and Law, 2010), the cost function can be redefine as

$$
J\left(\boldsymbol{\zeta}^{j+1}, \lambda\right)=\left\|\boldsymbol{S}^{j} \boldsymbol{\zeta}^{j+1}-\Delta \mathbf{P}_{c}^{j}\right\|_{2}^{2}+\lambda^{2}\left\|\sum_{k=1}^{j+1} \boldsymbol{\zeta}^{i}-\boldsymbol{\zeta}^{k, *}\right\|
$$

271 where $\lambda$ is the regularization parameter obtained from the $L$-curve method (Hansen, 1992). $\zeta^{j, *}$ 272 is a special vector related to damaged vector. When $j=0, \quad \zeta^{j, *}=0$, and when $j \geq 0$,

$$
\left(\zeta^{j, *}\right)_{i}=\left\{\begin{array}{ll}
0 & \text { if }\left(\sum_{k=1}^{j} \zeta^{j}\right)_{i} \geq 0 \\
\left(\sum_{k=1}^{j} \boldsymbol{\zeta}^{j}\right)_{i} & \text { if }\left(\sum_{k=1}^{j} \boldsymbol{\zeta}^{j}\right)_{i}<0
\end{array},(i=1,2, \cdots, n)\right.
$$

274 where $n$ is the number of parameters to be identified. Therefore, in the adaptive Tikhonov 275 regularization ( $\mathrm{Li}$ and Law, 2010), the damaged vector $\zeta^{j+1}$ can be obtained by minimizing the 276 cost function as

$$
\boldsymbol{\zeta}^{j+1}=\left(\left(\boldsymbol{S}^{j}\right)^{T} \boldsymbol{S}^{j}+\lambda^{2} \mathbf{I}\right)^{-1}\left(\left(\boldsymbol{S}^{j}\right)^{T} \Delta \mathbf{P}_{c}^{j}-\lambda^{2}\left(\sum_{k=1}^{j} \boldsymbol{\zeta}^{j}-\boldsymbol{\zeta}^{j, *}\right)\right)
$$

278 The quality of identified results can be gauged based on the gradient of the residual and penalty 
functions as

$$
\cos \theta=\frac{\left(\mathbf{r}^{j}\right)^{T} \cdot\left(\mathbf{A}^{j}\left(\left(\mathbf{A}^{j}\right)^{T} \mathbf{A}^{j}\right)^{-1}\left(\mathbf{A}^{j}\right)^{T} \mathbf{r}^{j}\right)}{\left\|\mathbf{r}^{j}\right\|_{2} \cdot\left\|\mathbf{A}^{j}\left(\left(\mathbf{A}^{j}\right)^{T} \mathbf{A}^{j}\right)^{-1}\left(\mathbf{A}^{j}\right)^{T} \mathbf{r}^{j}\right\|_{2}}
$$

281 where $\mathbf{A}^{j}=\left[\begin{array}{c}\boldsymbol{S}^{j} \\ \lambda^{j} \mathbf{I}\end{array}\right], \quad \mathbf{r}^{j}=\left[\begin{array}{c}\boldsymbol{\zeta}^{j} \\ -\lambda^{k}\left(\sum_{i=1}^{j} \boldsymbol{\zeta}^{j}-\boldsymbol{\zeta}^{j, *}\right)\end{array}\right]$. The solution is considered converged with iterations when angle $\theta$ approaches $90^{\circ}$.

The criterion of convergence in the iterative processes can be defined as

$$
\frac{\left\|\zeta^{j+1}-\zeta^{j}\right\|}{\left\|\zeta^{j+1}\right\|} \times 100 \% \leq \mathrm{Tol}
$$

where Tol is a small prescribed value and is taken equal to $2 \times 10^{-3}$ in the following studies unless otherwise specified.

The computation algorithm described above can be implemented in the following steps:

1) Set the initial value $\zeta^{0}$.

2) For the $j$ th step, the sensitivity matrix $S^{j}$ can be calculated from Eqs. (21) and (22).

3) Using the adaptive Tikhonov regularisation technique, Eq. (24) can be solved and the parameters $\zeta^{j+1}$ can be obtained.

4) Check the convergence using Eqs. (27) and (28). Repeat Steps 2 and 3 if it is not satisfactory.

\section{Numerical Simulations}

The track structure studied consists of finite length resting on 101 sleepers and ballasts underneath. Adjacent sleepers has a center-to-centre spacing of $0.6 \mathrm{~m}$. The middle 81 sleepers and ballast elements are considered in the studies to avoid any end effects in the dynamic analysis of the train-track system. The rail is modeled as Euler-Bernoulli beam discretized into 100 equal finite elements each with spring supports at two ends. The parameters of the vehicle and the track are shown in Table 1. The modal damping of the first two vibration modes of the rail are taken equal to 0.08. The irregularity amplitude of the rail corrugation is taken as $0.5 \mathrm{~mm}$. The vehicle moves from 
the first sleeper on the left to the last sleeper on the right. Data collected when the train is on top of the middle 81 sleepers are used for the identification.

The effect of measurement noise is simulated with a normally distributed random component with zero mean and a unit standard deviation added to the calculated acceleration response of the vehicle as

$$
\left\{\begin{array}{l}
\ddot{\boldsymbol{y}}_{v 1}^{\text {polluted }}=\ddot{\boldsymbol{y}}_{v 1}^{\text {calculated }}+E_{p} \times \mathbf{N}_{\text {oise }}^{1} \times \operatorname{var}\left(\ddot{\boldsymbol{y}}_{v 1}^{\text {calculated }}\right) \\
\ddot{\boldsymbol{y}}_{v 2}^{\text {polluted }}=\ddot{\boldsymbol{y}}_{v 2}^{\text {calculated }}+E_{p} \times \mathbf{N}_{\text {oise }}^{2} \times \operatorname{var}\left(\ddot{\boldsymbol{y}}_{v 2}^{\text {calculated }}\right)
\end{array}\right.
$$

where $\ddot{\boldsymbol{y}}_{v 1}^{\text {polluted }}$ and $\ddot{\boldsymbol{y}}_{v 2}^{\text {polluted }}$ are vectors of polluted "measured" acceleration response; $\ddot{\boldsymbol{y}}_{v 1}^{\text {calculated }}$ and $\ddot{\boldsymbol{y}}_{v 2}^{\text {calculated }}$ are the calculated acceleration response of the vehicle; $E_{P}$ is the noise level; $\mathbf{N}_{\text {oise }}^{1}$ and $\mathbf{N}_{\text {oise }}^{2}$ are two different normal random vectors with zero mean and unit variance; $\operatorname{var}(\bullet)$ is the standard deviation of the calculated acceleration response.

The relative error of the identified stiffness can be defined as

$$
\text { Relative Error }=\left|\frac{\boldsymbol{k}^{\text {id }}-\boldsymbol{k}^{\text {true }}}{\boldsymbol{k}^{\text {true }}}\right| \times 100 \%
$$

where $\boldsymbol{k}^{\text {id }}$ and $\boldsymbol{k}^{\text {true }}$ are the identified and true stiffness parameter vectors, respectively.

\section{Identification of Winkler elastic foundation}

\section{Single foundation settlement}

\section{Case 1: Effect of moving speed}

The scenarios with the vehicle moves at a constant speed of $10 \mathrm{~m} / \mathrm{s}, 30 \mathrm{~m} / \mathrm{s}$ and $50 \mathrm{~m} / \mathrm{s}$ are studied. The sampling rate in the dynamic analysis is $5000 \mathrm{~Hz}$. Assuming that there is only one zone with foundation settlement in the track substructure which is $12 \mathrm{~m}$ long with the mid-length of the zone at 20 meter from the left end of the system. The magnitude of stiffness reduction $\alpha$ is set equal to 0.2. The true foundation stiffness loss is shown in Figure 2. The foundation stiffness vector $\zeta$ at the beginning of iteration is set equal to null. The identified results with $0 \%, 5 \%$ and $10 \%$ noise 
level are shown in Figure 3. Accurate identified results can be obtained when there is no noise effect.

324 This verifies the accuracy of the proposed inverse analysis. However, when measurement noise is

325 involved, a lower vehicle travelling speed may lead to more accurate identified result. Table 2

326 shows that the parameter $\cos \theta$ is very close to zero when there is noise effect. This indicates that

327 the identified results cannot be further improved with more iteration as indicated by the property of

$328 \cos \theta$ in Eq. (27). It is also noted that the parameter $\cos \theta$ is relative large when there is no noise

329 effect indicating further improvement in the identified results can be made probably with a smaller

330 threshold of acceptance in Eq. (28). However, the computation stops when the convergence

331 threshold is achieved.

\section{Case 2: Effect of sampling rate}

333 The sampling rate of $2000 \mathrm{~Hz} 、 5000 \mathrm{~Hz}$ and $10000 \mathrm{~Hz}$ are studied to check on the effect different

334 sampling rates. The vehicle moves at $30 \mathrm{~m} / \mathrm{s}$. Other parameters are the same as for last study. Results

335 in Figures 3(b) and 4 show that the accuracy of identification increases with the sampling rate.

336 When the sampling rate is $2000 \mathrm{~Hz}$, the location of the settlement zone can be identified

337 successfully but with a poor estimate on the magnitude of damage when there is measurement noise.

338 Therefore a higher sampling rate of $10000 \mathrm{~Hz}$ is adopted in the following studies.

\section{Multiple foundation settlement}

340 The foundation settlement is associated with flexibility at the same location. Three zones of settlement are considered, and the three zones of foundation flexibility are assumed overlapping in the track substructure. The first one has a linear distribution starting at $12 \mathrm{~m}$ from the left end of the system considered with a length of $8 \mathrm{~m}$. The second and third ones have parabolic distributions centered at $20 \mathrm{~m}$ and $27 \mathrm{~m}$ from the left side respectively with a length of $12 \mathrm{~m}$ or $20 \mathrm{~m}$, respectively. 
$\alpha$, at mid-length of the three zones are respectively $10 \%, 20 \%$ and $18 \%$. The vehicles is assumed to

move on the track at $30 \mathrm{~m} / \mathrm{s}$ and the sampling rate is $10000 \mathrm{~Hz}$. Results in Figure 6 show that the distribution of stiffness changes can be identified successfully after 14, 30, and 24 iterations with $0 \%, 5 \%$ and $10 \%$ measurement noise. The relative error of the identified results is smaller at lower noise level with the maximum error of $7.44 \%$ at spring 32 when there is $10 \%$ measurement noise.

\section{Condition identification of rail fasteners}

The local damage due to a loosened rail fastener is simulated as a reduction of the corresponding elemental connection spring stiffness between the rail and sleeper. Multiple damages in the rail fasteners are simulated with $50 \%, 25 \%, 20 \%, 10 \%$ and $12 \%$ stiffness loss at the springs $18,25,46$, 67 and 81 . The sampling rate is $10000 \mathrm{~Hz}$ and the moving speed is $30 \mathrm{~m} / \mathrm{s}$. Other parameters are the same as for last study.

The identification results with $1 \%, 5 \%$ and $10 \%$ noise level are shown in Figure 7. Damage in the rail fasteners can be identified successfully even with $10 \%$ noise level. Figure 8 shows that the value of $\cos \theta$ approaches a minimum after only a few iterations indicating convergence of the identified results. Such convergence is particularly noted in the scenario with $10 \%$ noise level with the adaptive Tikhonov regularization. Results converged after 12, 14 and 92 iterations with the maximum relative error of $1.6 \%, 3.0 \%$ and $5 \%$ as shown in Figure 8 for the scenarios with $1 \%, 5 \%$ and $10 \%$ noise level respectively.

\section{Identification of ballast damage}

Multiple local zones of loosely compacted ballast are assumed in the track substructure. These zones are modeled with $8 \%, 10 \%, 15 \%$ and $20 \%$ stiffness loss at the springs $21,36,53$ and 75 . Other parameters are the same as those in last study. The identification results are shown in Figure 10. The damage location can be identified successfully for all noise level studied. However, the 
identified damage extent is satisfactory only when the noise level is equal or less than $5 \%$. Figure 11 shows the evolution of the converging results. Results converged after 9, 19 and 41 iterations with the maximum relative error of $0.6 \%, 1.0 \%$ and $10 \%$ as shown in Figure 12 for the scenarios with $1 \%, 5 \%$ and $10 \%$ noise level respectively.

\section{Assessment of the track substructure including all types of defects}

\section{Identification with different noise levels}

Different types of defects may coexist in the track substructure. The different defects studied in this section include: (a) two damaged rail fasteners with 15\% and 10\% stiffness loss at springs 25 and 67; (b) two ballasts units with 20\% and 15\% stiffness loss at springs 41 and 53; and (c) one zone of foundation flexibility as described in the section "Single foundation settlement". The damage vector contains the stiffness change in units 11 to 91 with 243 unknown spring stiffness changes. Measurements with $0 \%, 1 \%$ and $5 \%$ noise are studied.

The identified results and the associated relative errors are shown in Figures 13 and 14. The local stiffness changes can be identified accurately when there is no noise effect as shown in Figure 13(a). When there is only $1 \%$ noise effect, rail fasteners 41 and 53 are identified incorrectly with the damage information from the stiffness change in the ballast unit transmitted into the rail fasteners. The location of damage in the rail fasteners and the ballast cannot be correctly identified with 5\% noise effect. This is because of the coupling of the spring stiffnesses from the rail fasteners, the ballast and the foundation as they are modeled in series. The identification of stiffness from the rail fasteners, damaged ballast and foundation settlement has been greatly affect by this coupling when measurement noise is involved. However, the zone of foundation defect can still be identified satisfactory with $5 \%$ noise effect but with large relative error up to $18 \%$. The number of iteration required for convergence is 28, 41 and 39 as noted in Figure 15 for the scenarios with $0 \%, 1 \%$ and $5 \%$ measurement noise respectively.

\section{Effect of wheel flat}


The last study is conducted again in this section including the effect of wheel flat with the parameters given in Table 1. Other parameters are the same as those in last study. The identification results for the scenarios of $0 \%$ and 5\% noise level after 45 and 92 iterations respectively are shown in Figure 16. Both the damage location and extent are noted not able to be identified satisfactory using the proposed approach. It may be concluded that the identification of the track substructure with coupling components is not feasible with a detailed discrete model as shown in Figure 1.

\section{Identification of an equivalent track substructure}

The coupling effect of the different springs in each unit is further studied with the track and substructure modeled by equivalent units each including the rail fasteners, the ballast and the foundation. The equivalent stiffness of the $i$ th element can be written as

$$
k_{e i}=\left(\frac{1}{k_{p i}}+\frac{1}{k_{s i}}+\frac{1}{k_{f i}+2 k_{w i}}\right)^{-1}
$$

The stiffness and damping of the equivalent units are computed for the track model studied in the last two sections, and the simplified model is shown in Figure 17. The boundaries of the equivalent unit at the bottom and at the two adjacent ballast are assumed fixed. The mass of the sleeper and the ballast are ignored and there is no interaction between two adjacent elements. The equivalent damping of the $i$ th element can be obtained similarly to the equivalent stiffness. The stiffness reduction of the $i$ th element can be obtained as

$$
\zeta_{i}=\left(1-\frac{k_{e i}}{k_{e 0}}\right) \times 100
$$

where $k_{e 0}$ is the equivalent stiffness of the intact element.

All the parameters are the same as for the section "Identification with different noise level". The identified results for the scenarios with $0 \%, 1 \%$ and $5 \%$ noise in the measurement are shown in Figure 18. Comparison with Figure 13 show that the identification results from equivalent track model is slightly better than those from the refined model when there is measurement noise. However, the identified foundation parameters from the equivalent model is not very distinct when 
there is noise effect.

420

421

\section{Discussions}

(a) There may be a concern with the higher sampling rate of $10000 \mathrm{~Hz}$ and a low speed of $30 \mathrm{~m} / \mathrm{s}$ as adopted in the above studies. This combination of parameters would mean 200 data will be collected within the time duration when the vehicle moves over the distance of $0.6 \mathrm{~m}$ from one sleeper to another. All the numerical results suggest more data included in the analysis could cancel the effect due to measurement noise giving better results. The best combination studied in this paper is sampling at $5000 \mathrm{~Hz}$ at vehicle speed at $10 \mathrm{~km} / \mathrm{s}$ gives 300 data within this time duration. This combination may be changed to have 400 data or 600 data which would mean sampling at $10000 \mathrm{~Hz}$ with the vehicle moving at $15 \mathrm{~m} / \mathrm{s}$ and $10 \mathrm{~m} / \mathrm{s}$ respectively. These speeds are equivalent to $54 \mathrm{~km} / \mathrm{s}$ and $36 \mathrm{~km} / \mathrm{s}$ which is normal when the train vehicle moves over section of track under maintenance operation according to current safety practices.

(b) The complexity of the train vehicle (e.g. with 2-DOFs) and its mass have been reported (Bu et al., 2006) to have effect on the condition assessment of bridge deck using measurement on deck. This study, however, addresses the problem with realistic standard vehicle recognized by all other researchers. Any change with parameters of the vehicle would lead to unrealistic results and therefore no attempt has been made to study these two factors.

\section{Conclusions}

A system identification methodology is proposed for the condition assessment of the railway track and its substructure with measurement from the moving vehicle. Finite element model with discrete elements representing different components of the structure is formulated, and the solution of the identification equation is solved with the adaptive regularization technique. Numerical studies show that all the damage parameters can be identified accurately when there is no measurement noise. But when measurement noise is included, more data collected from using a higher sampling rate and a lower moving speed can yield satisfactory results in the scenario of having a single damage. 
When there are damages of different types to be identified, the identified results are not reliable as the discrete components in the different layers of the track structure are conne4cted in series and their changes in stiffness are coupling with each other. The identification with an equivalent track model, however, can give slightly better results in the case with noisy measurement.

\section{References}

[1] H.F. Lam, M.T. Wong and Y.B. Yang, A feasibility study on railway ballast damage detection utilizing measured vibration of in situ concrete sleeper, Engineering Structures, 2012, 45: 284-298.

[2] H.F. Law, S.A. Alabi and J.-H. Yang, Identification of rail-sleeper-ballast system through time-domain Markov chain Monte Carlo-based Bayesian approach, Engineering Structures, 2017, 140: 421-436.

[3] H. Ishii, Y. Fujino, Y. Mizuno and K. Kaito, The study of train intelligent monitoring system using acceleration of ordinary trains, Proceedings of the 1st Asia-Pacific Workshop on Structural Health Monitoring, 4-6 December, 2006, Yokohama, Japan.

[4] Y. Mizuno, Y. Fujino, K. Kataoka and Y. Matsumoto, Development of a mobile sensing unit and its prototype implementation, Tsinghua Science and Technology, 2008, 13: 223-227.

[5] P.F. Weston, C.S. Ling, C.J. Goodman, C. Roberts, P. Li and R.M. Goodall, Monitoring lateral track irregularity form in-service railway vehicles, Proceedings of Institution of Mechanical Engineers, Part F: Journal of Rail and Rapid Transit, 2007, 221: 89-100.

[6] C. With and A. Bodare, Evaluation of track stiffness with a vibrator for prediction of train-induced displacement on railway embankments, Soil Dynamics and Earthquake Engineering, 2009, 29(8): 1187-1197.

[7] D. Cantero and B. Basu, Railway infrastructure damage detection using wavelet transformed acceleration response of traversing vehicle, Structural control and health monitoring, 2015, 22(1), 62-70.

[8] P. Salvador, V. Naranjo, R. Insa and P. Teixeira, Axlebox accelerations: their acquisition and 
time-frequency characterization for railway track monitoring purposes, Measurement, 2016, 82: 301-312.

[9] G. Lederman, S, Chen, J. Garrent, J. Kovacevic, H.Y.Noh and J. Bielak, Track-monitoring from the dynamic response of an operational train. Mechanical Systems and Signal Processing, $2017,87,1-16$.

[10] M. Oregui, S. Li, A. Nunez, Z. Li, R. Carroll and R.Dollevoet, Monitoring bolt tightness of rail joints using axle box acceleration measurements. Structural Control and Health Monitoring, 24: $10.1002 /$ stc. 1848 .

[11] C.S. Li, S.H. Luo, C. Cole and M. Spiryagin, An overview: modern techniques for railway vehicle on-board health monitoring systems, Vehicle System Dynamics, 2017, 55(7): 1045-1070.

[12] W. Zhai and Z. Cai, Dynamic interaction between a lumped mass vehicle and a discretely supported continuous rail track. Computers \& Structures, 1997, 63(5): 987-997.

[13] R. U. A. Uzzal, W. Ahmed and S. Rakheja, Dynamic analysis of railway vehicle-track interactions due to wheel flat with a pitch-plane vehicle model, Journal of Mechanical Engineering, 2008, 39(2): 86-94.

[14] S. Mohammadzadeh, M. Esmaeili and M. Mehrali, Dynamic response of double beam rested on stochastic foundation under harmonic moving load, International Journal for Numerical and Analytical Methods in Geomechanics, 2014, 38: 572-592.

[15] Y.B. Yang and J.D. Yau, Vehicle bridge interaction element for dynamic analysis, Journal of Structural Engineering ASCE, 1997, 123(11): 1512-1518

[16] G. Savini, A Numerical Program for Railway Vehicle-Track-Structure Dynamic Interaction using a Modal Substructuring Approach, University of Bologna Digital Library, 2010.

[17] S.S. Law, K. Zhang and Z.D. Duan, Structural damage detection from coupling forces between substructures under support excitation, Engineering Structures, 2010, 32: 2221-2228.

[18] X.Y. Li and S.S. Law, Adaptive Tikhonov regularization for damage detection based on 
nonlinear model updating, Mechanical Systems and Signal Processing, 2010, 24(6): 1646-1664.

498

499

500

501

502

503

504

505 506

507

508

509

510

511

512

513

514

515

516

517

518

[19] A. Kacar, H. Tugba Tan and M.O. Kaya, Free vibration analysis of beams on variable Winkler elastic foundation by using the differential transform method, Mathematical and Computational Applications, 2011, 16(3):773-783.

[20] C. Vale and R. Calcada, A dynamic vehicle-track interaction model for predicting the track degradation process, Journal of Infrastructure Systems ASCE, 2014, 20(3), 04014016.

[21] T.X. Wu and D.J. Thompson, A hybrid model for the noise generation due to railway wheel flats, Journal of Sound and Vibration, 2002, 251(1):115-139

[22] K.J. Bathe, Finite Element Procedures in Engineering Analysis, New Jersey: Prentice Hall, 1982.

[23] K. Henchi, M. Fafard, M. Talbot, and G. Dhatt, An efficient algorithm for dynamic analysis of bridges under moving vehicles using a coupled modal and physical components approach, Journal of sound and vibration, 1988, 212(4):663-683.

[24] P.C. Hansen, Analysis of discrete ill-posed problems by means of the L-curve, SIMA Review, 1992, 34 (4): 561-580.

[25]K. Liu, S.S. Law, X.Q. Zhu and Y. Xia (2014) Explicit form of an implicit method for inverse force identification. Journal of Sound and Vibration, 2014, 333(3): 730-744.

[26]J.Q. Bu, S.S. Law and X.Q. Zhu (2006). Innovative bridge damage assessment from dynamic response of a passing vehicle. Journal of Engineering Mechanics, ASCE. 132(12), 1372-1379. 
Vehicle model parameters (Vale Track model parameters and Calcada, 2013) (Zhai and Cai, 1997)

\begin{tabular}{ll}
\hline$m_{v 1}=5600 \mathrm{~kg}$ & $m_{r}=51.5 \mathrm{~kg} \cdot \mathrm{m}^{-1}$ \\
$m_{v 2}=2003 \mathrm{~kg}$ & $E I=4.2 \times 10^{6} \mathrm{Nm}^{2}$ \\
$k_{v}=4.80 \times 10^{6} \mathrm{Nm}^{-1}$ & $L_{s}=0.6 \mathrm{~m}$ \\
$c_{v}=1.08 \times 10^{5} \mathrm{Nsm}^{-1}$ & $m_{s i}=273 \mathrm{~kg}$ \\
$\mathrm{~K}_{\mathrm{H}}=1.3964 \times 10^{9} \mathrm{Nm}^{-1}$ & $m_{b i}=683 \mathrm{~kg}$ \\
$D_{f}=0.4 \mathrm{~mm}$ & $k_{p i}=1.2 \times 10^{8} \mathrm{Nm}^{-1}$ \\
$L_{f}=52 \mathrm{~mm}$ & $c_{p i}=1.24 \times 10^{5} \mathrm{Nsm}^{-1}$ \\
$R_{w}=420 \mathrm{~mm}$ & $k_{b i}=2.4 \times 10^{8} \mathrm{Nm}^{-1}$ \\
& $c_{b i}=5.88 \times 10^{4} \mathrm{Nsm}^{-1}$ \\
& $k_{f i}=6.5 \times 10^{7} \mathrm{Nm}^{-1}$ \\
& $c_{f i}=3.12 \times 10^{4} \mathrm{Nsm}^{-1}$ \\
& $k_{w i}=7.84 \times 10^{7} \mathrm{Nm}^{-1}$ \\
& $c_{w i}=8.0 \times 10^{4} \mathrm{Nsm}^{-1}$ \\
\hline
\end{tabular}




\begin{tabular}{cccccccccc}
\hline \multirow{2}{*}{ Scenarios } & \multicolumn{3}{c}{$10 \mathrm{~m} / \mathrm{s}$} & \multicolumn{3}{c}{$30 \mathrm{~m} / \mathrm{s}$} & \multicolumn{3}{c}{$50 \mathrm{~m} / \mathrm{s}$} \\
\cline { 2 - 10 } & Nil & $5 \%$ & $10 \%$ & Nil & $5 \%$ & $10 \%$ & Nil & $5 \%$ & $10 \%$ \\
\hline Parameter $\lambda$ & $5.77 \mathrm{e}-6$ & $5.79 \mathrm{e}-6$ & $5.81 \mathrm{e}-6$ & $8.25 \mathrm{e}-6$ & $2.19 \mathrm{e}-5$ & $2.78 \mathrm{e}-5$ & $1.84 \mathrm{e}-5$ & $3.29 \mathrm{e}-5$ & $3.73 \mathrm{e}-5$ \\
$\cos \theta$ & 0.997 & 0.023 & 0.014 & 0.812 & 0.052 & 0.051 & 0.690 & 0.126 & 0.053 \\
Max. RE. (\%) & $8.40 \mathrm{e}-5$ & -3.24 & -8.03 & $4.19 \mathrm{e}-3$ & -5.93 & -11.99 & $6.54 \mathrm{e}-5$ & -7.01 & -13.29 \\
Iteration No. & 9 & 16 & 15 & 16 & 33 & 37 & 8 & 98 & 92 \\
\hline
\end{tabular}

522 Noted: Max. RE. denotes the maximum relative error for all the elements; Iteration No. denotes the number 
Table 3 - Identification results with different sampling rate when moving at $30 \mathrm{~m} / \mathrm{s}$

\begin{tabular}{ccccccc}
\hline \multirow{2}{*}{ Scenario } & \multicolumn{3}{c}{$2000 \mathrm{~Hz}$} & \multicolumn{3}{c}{$10000 \mathrm{~Hz}$} \\
\cline { 2 - 7 } & Nil & $5 \%$ & $10 \%$ & Nil & $5 \%$ & $10 \%$ \\
\hline Parameter $\lambda$ & $1.84 \mathrm{e}-5$ & $1.17 \mathrm{e}-5$ & $2.27 \mathrm{e}-5$ & $3.83 \mathrm{e}-6$ & $6.68 \mathrm{e}-5$ & $4.48 \mathrm{e}-6$ \\
$\cos \theta$ & 0.829 & 0.041 & 0.104 & 0.814 & 0.026 & 0.040 \\
Max. RE. (\%) & $4.8 \mathrm{e}-3$ & -8.45 & -13.78 & $4.64 \mathrm{e}-3$ & -0.73 & -3.42 \\
Iter. No. & 16 & 67 & 95 & 15 & 13 & 78 \\
\hline
\end{tabular}

526

Note: Results for $5000 \mathrm{~Hz}$ sampling rate is referred to Table 2. 


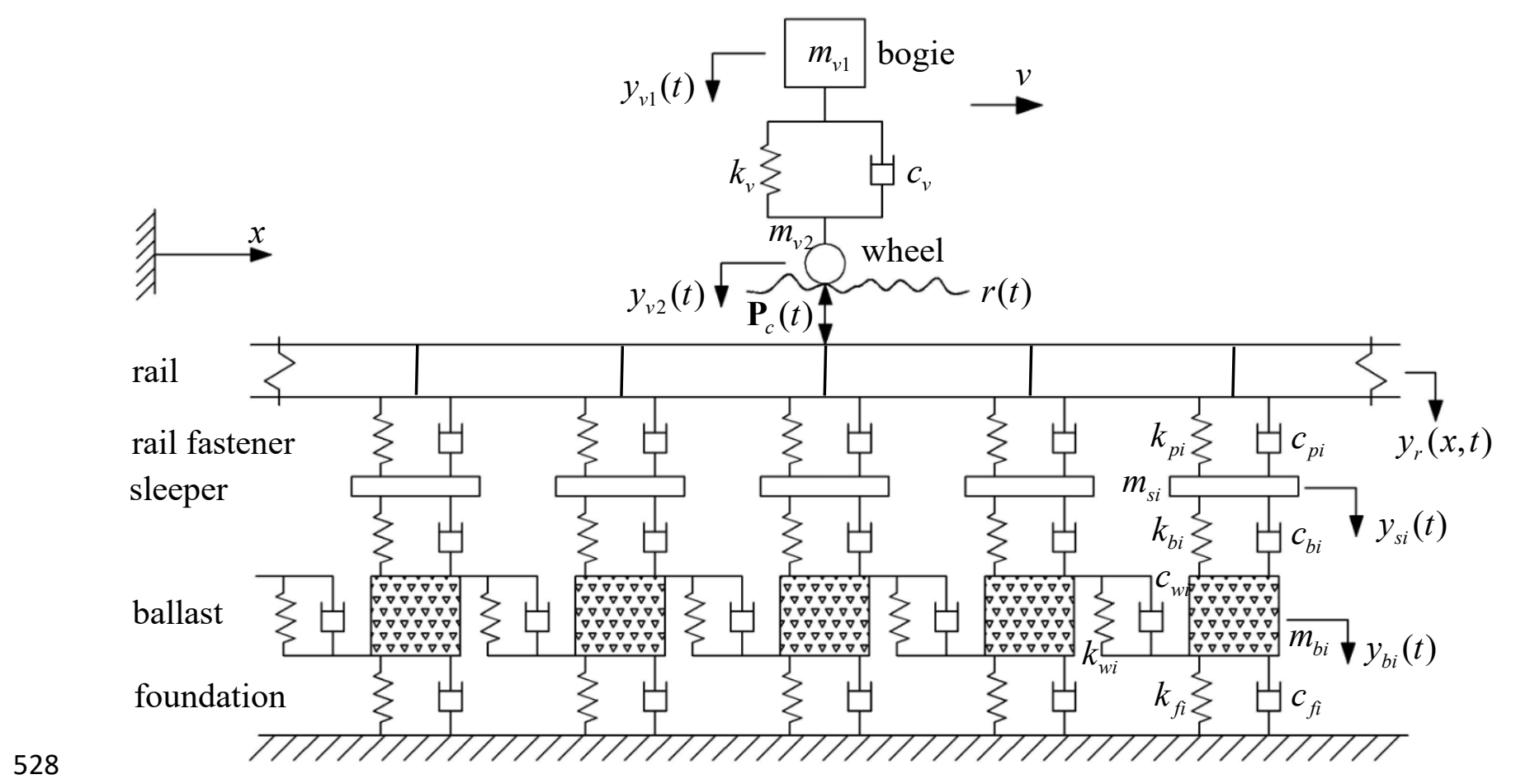

Figure 1: Train vehicle and rail track system model
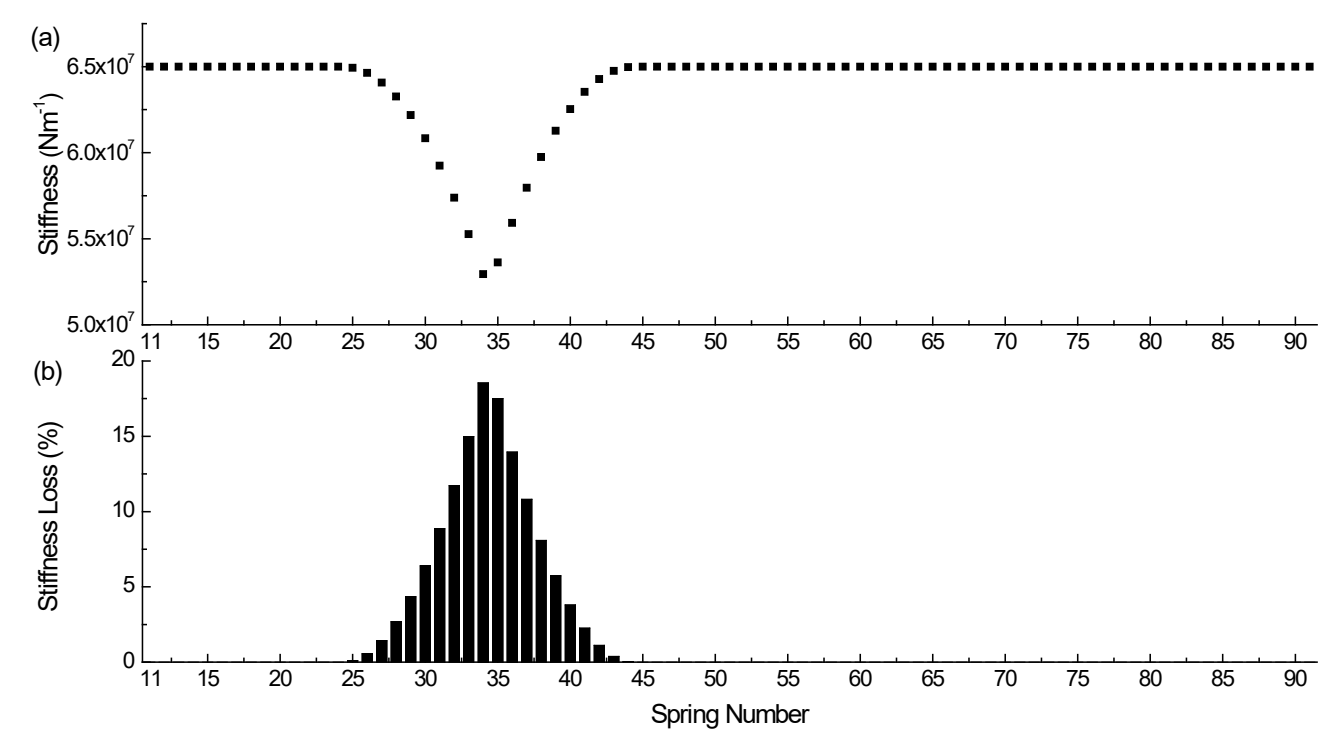

Figure 2: True foundation stiffness loss in each element with single settlement zone

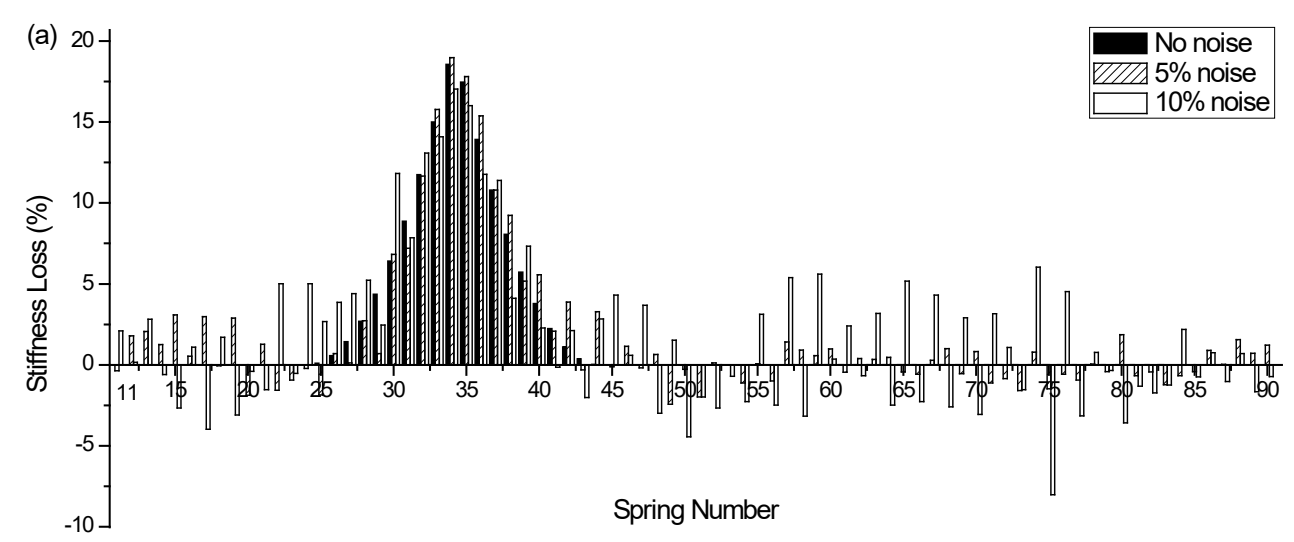



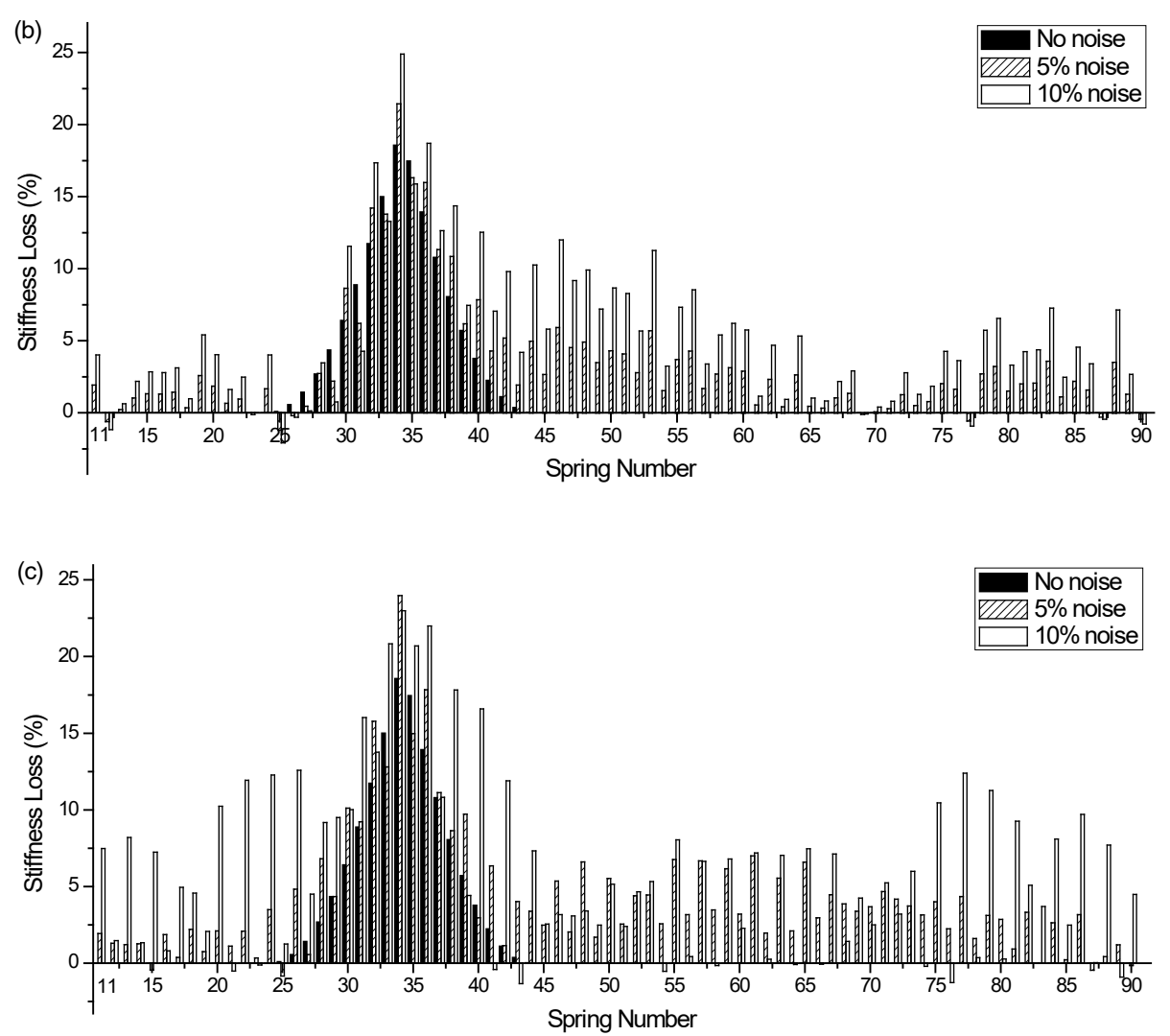

Figure 3: Identification results at different moving speed: (a) $10 \mathrm{~m} / \mathrm{s}$; (b) $30 \mathrm{~m} / \mathrm{s}$; (c) $50 \mathrm{~m} / \mathrm{s}$

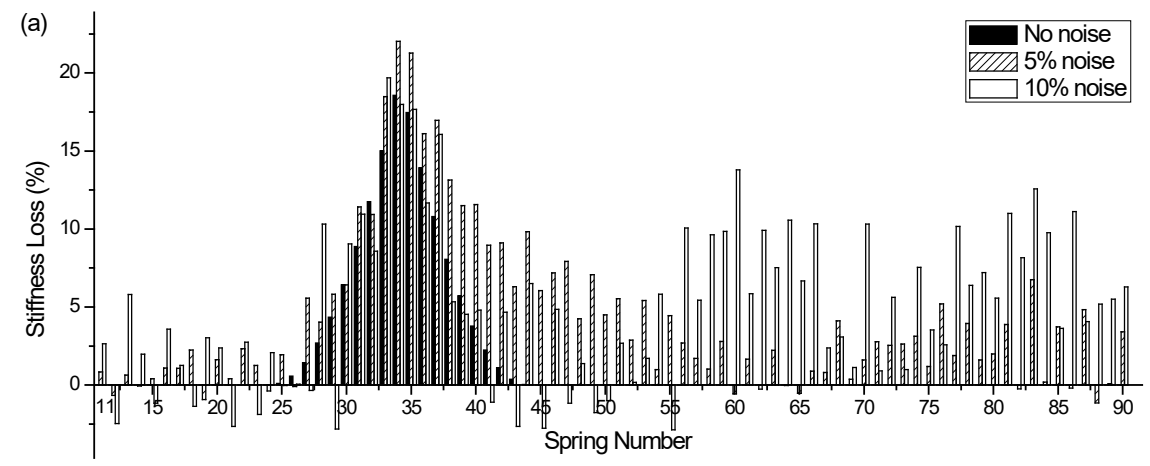

(b)

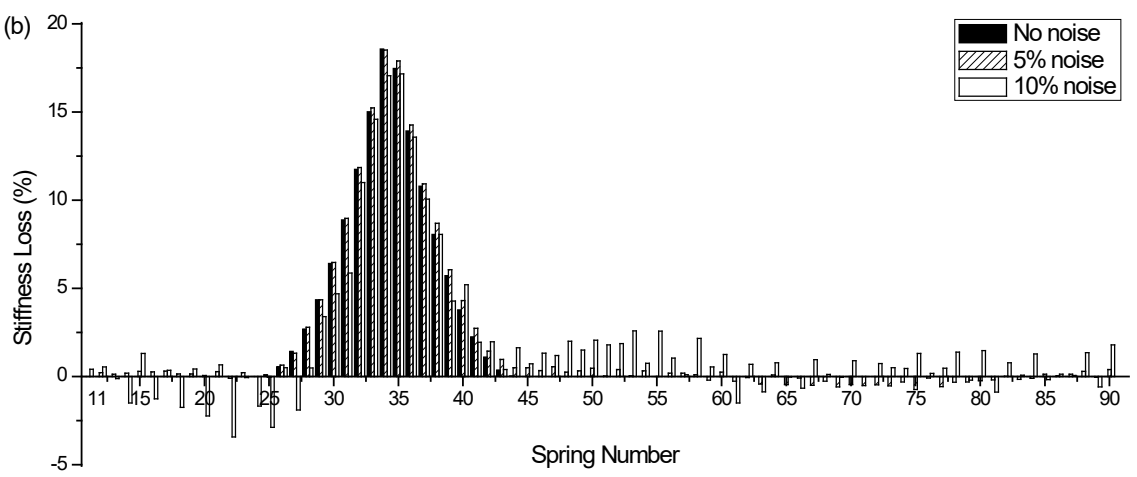

Figure 4: Identification results with different sampling rate: (a) 2000HZ; (b) 10000HZ 


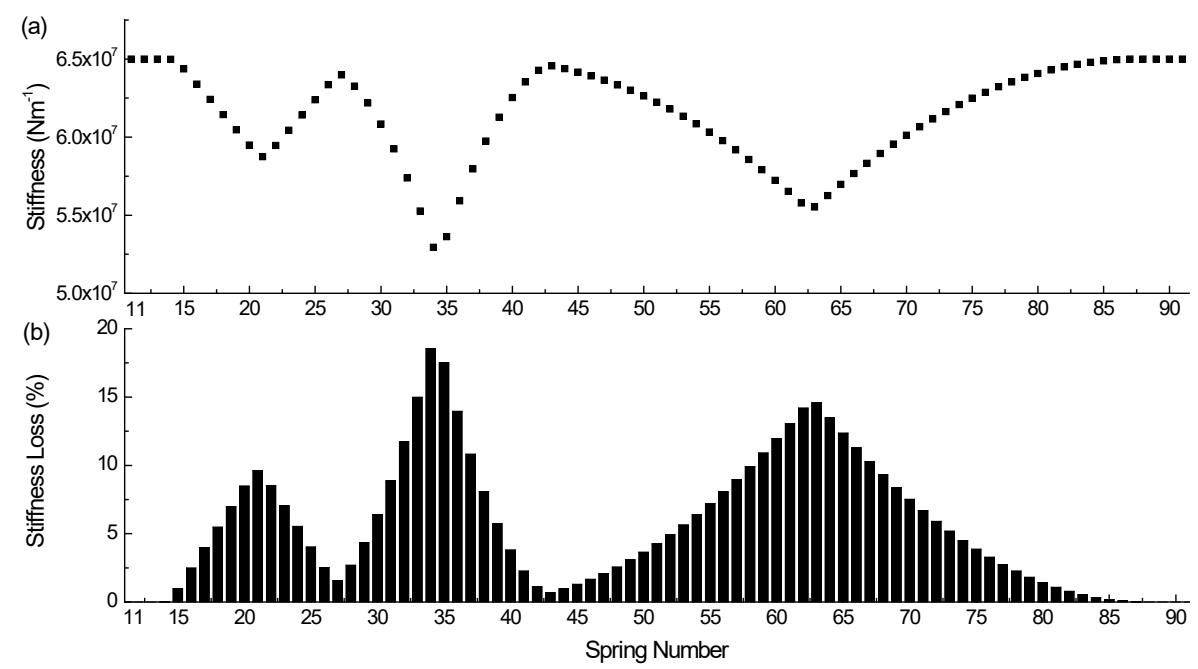

Figure 5: True foundation stiffness loss in each element with multiple settlement zones

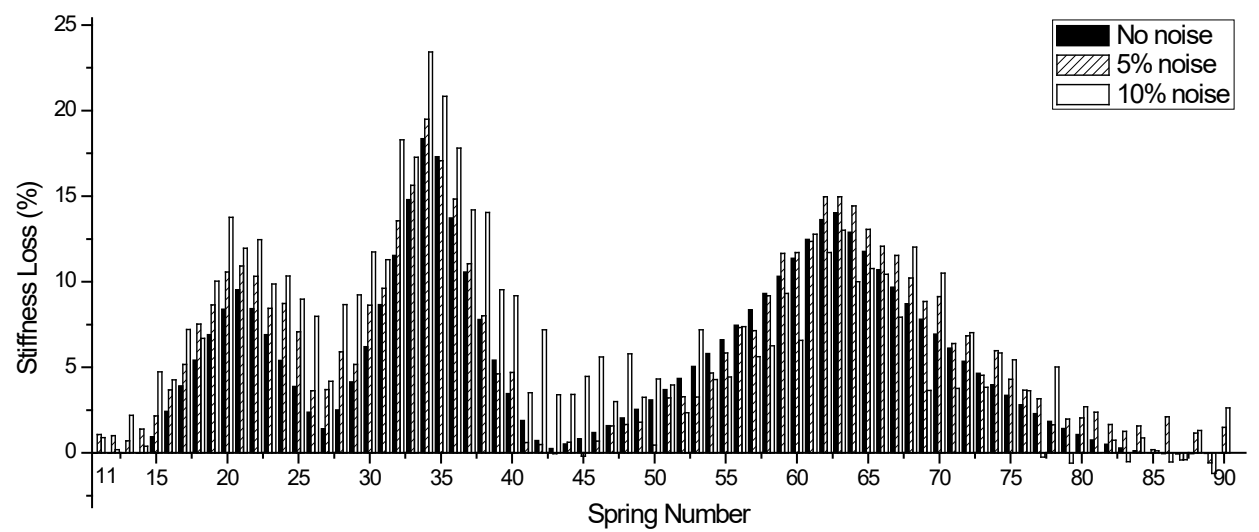

Figure 6: Multiple damage identification results

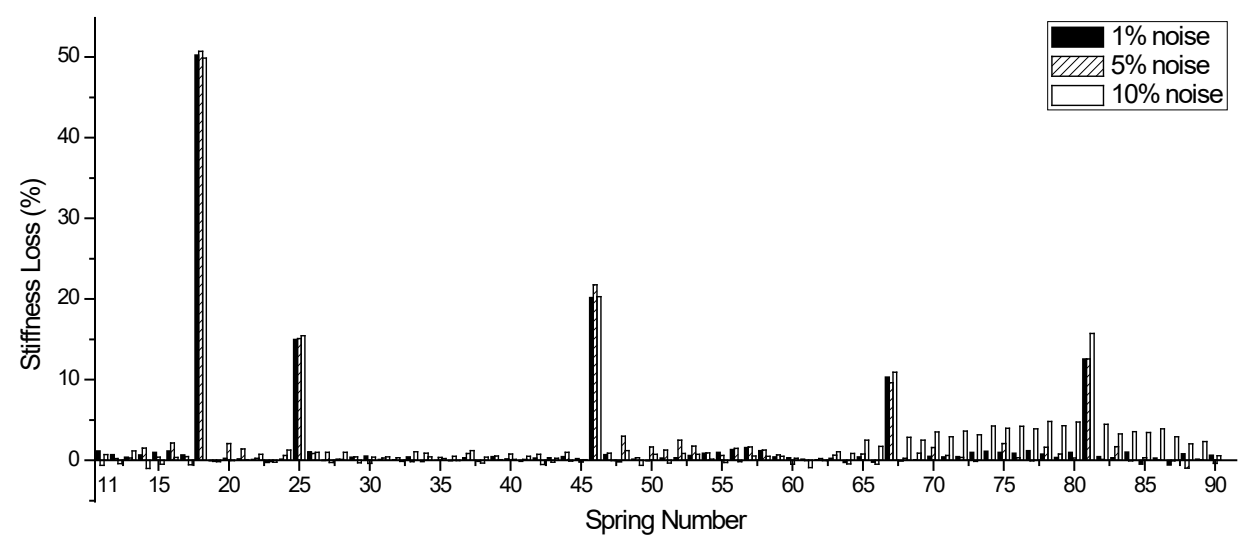

Figure 7: Identification results of rail fastening 

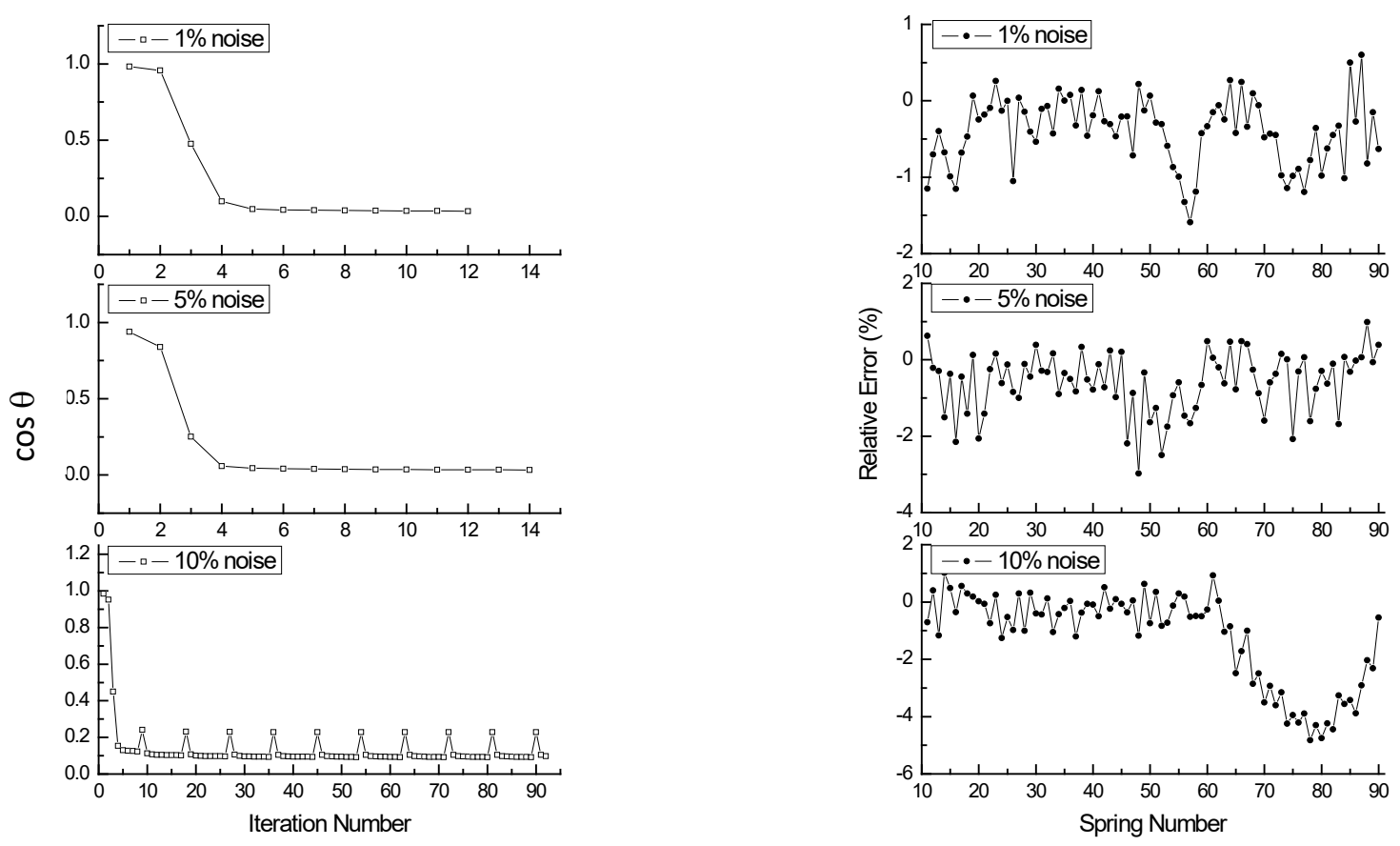

Figure 8: Evolution of $\cos \theta$ value

Figure 9: Relative Error

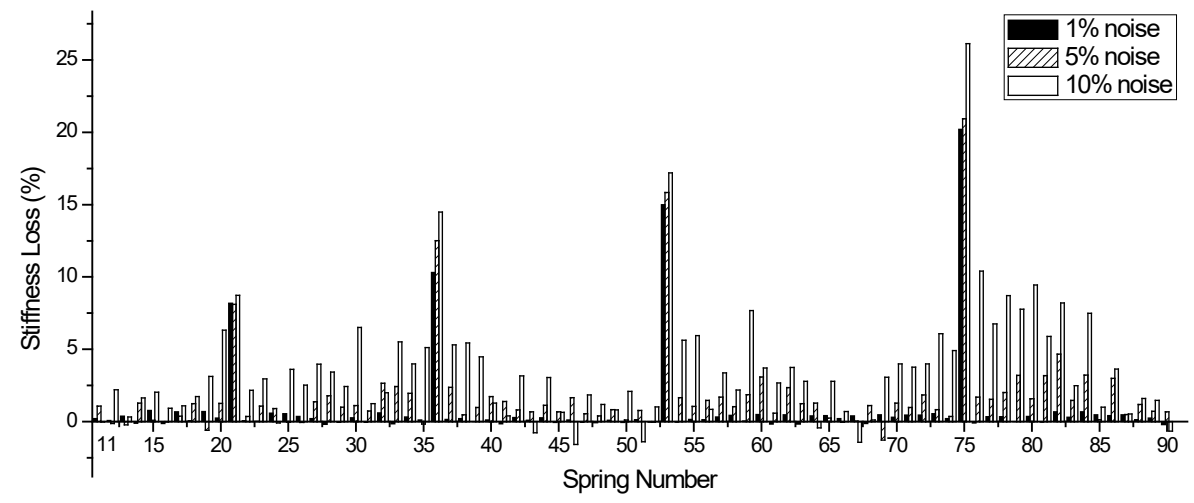

Figure 10: Identification results of damaged ballast
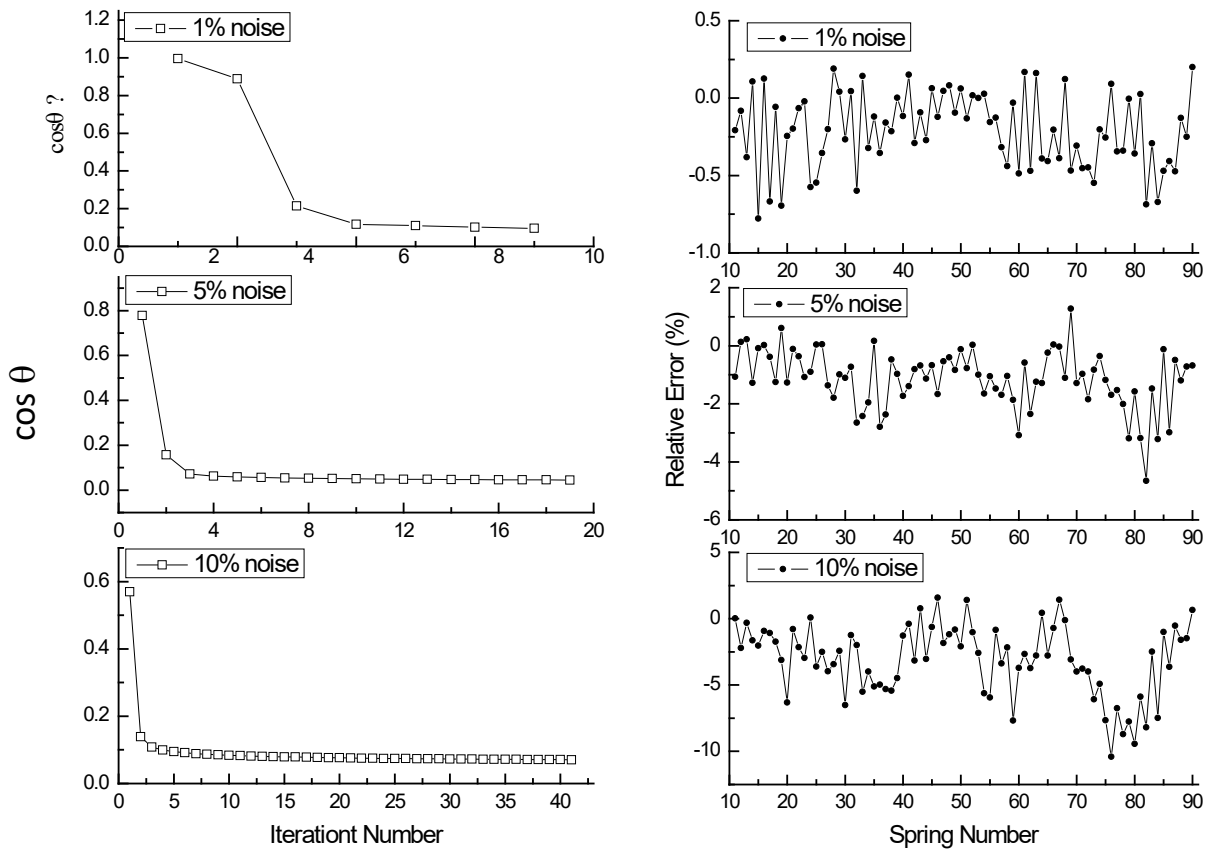

Figure 11: Evolution of $\cos \theta$ value

Figure 12: Relative Error 


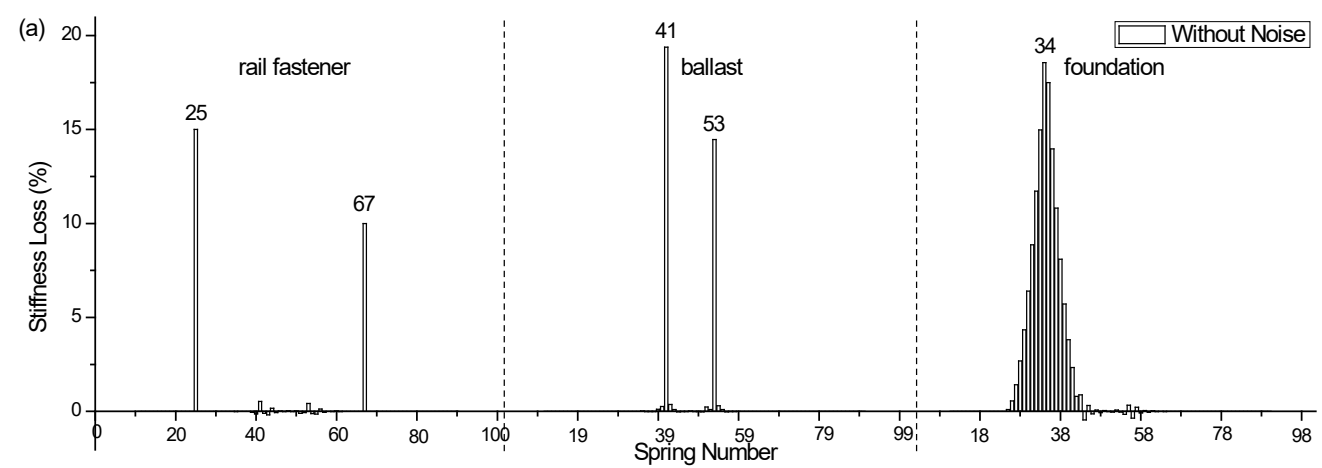

(a) Identified results from measurements without noise

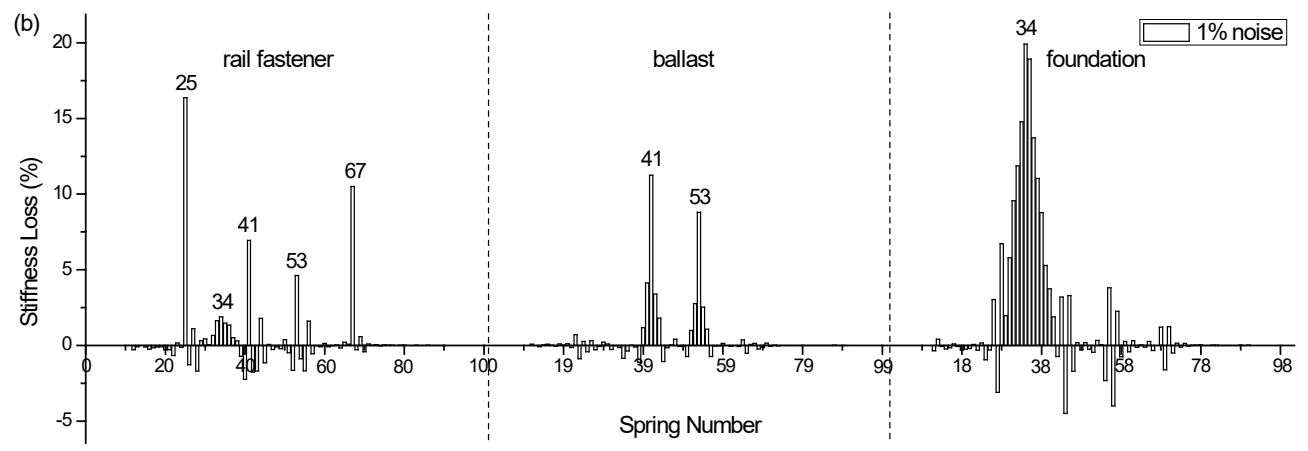

(b) Identified results with $1 \%$ measurements noise

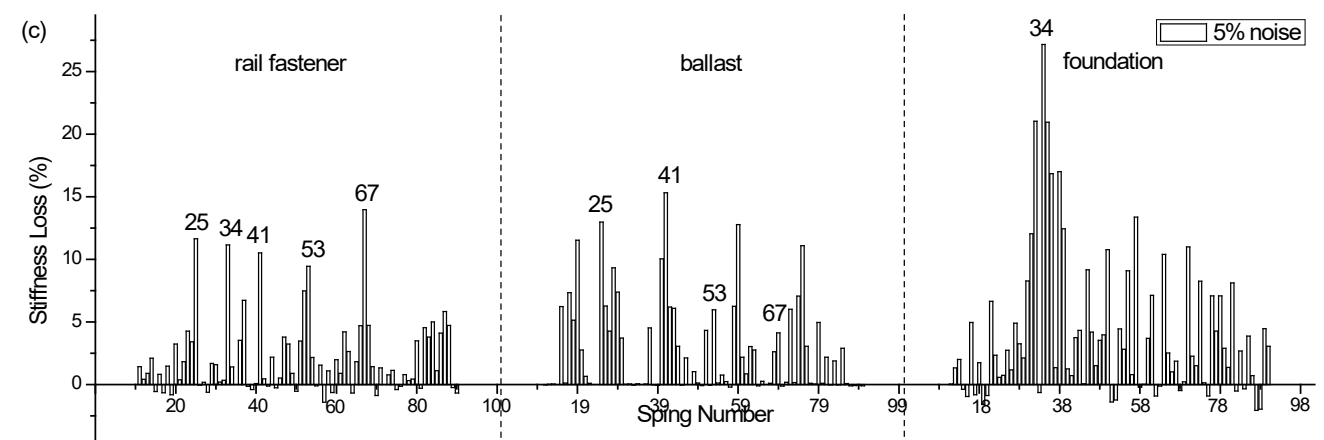

(b) Identified results with $5 \%$ measurements noise

Figure 13: Identification results of the whole substructure

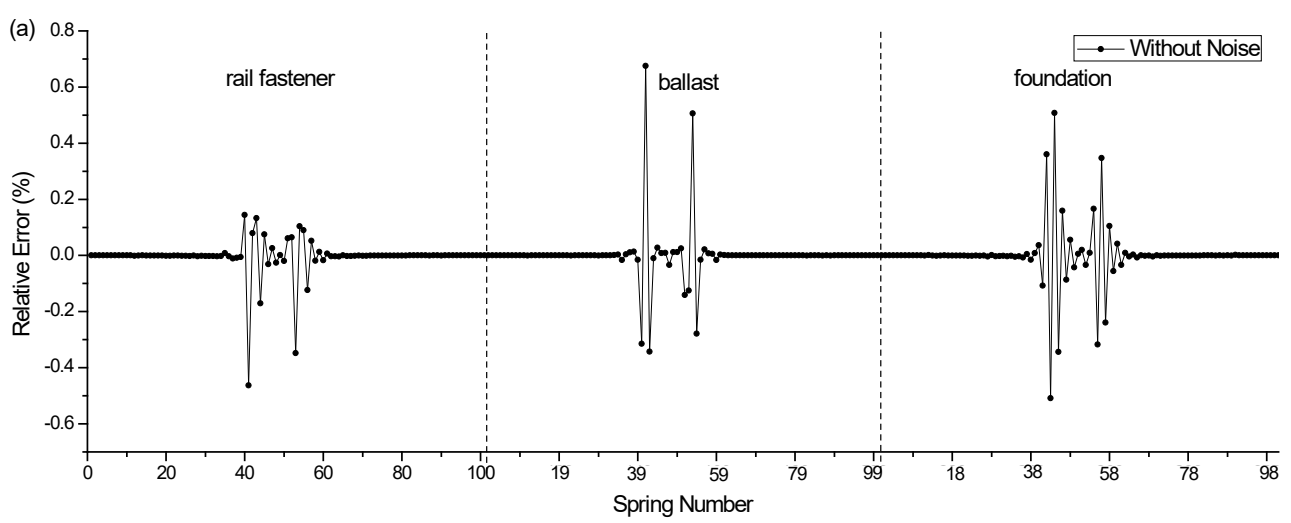

(a) Identified results without the measurements noise 


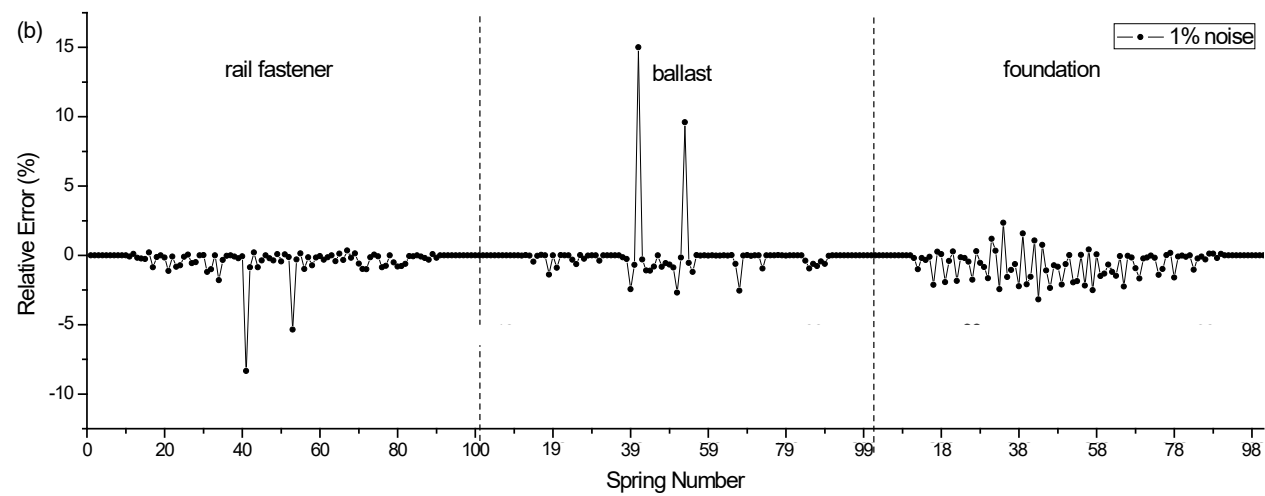

567

(b) Identified results with $1 \%$ measurements noise

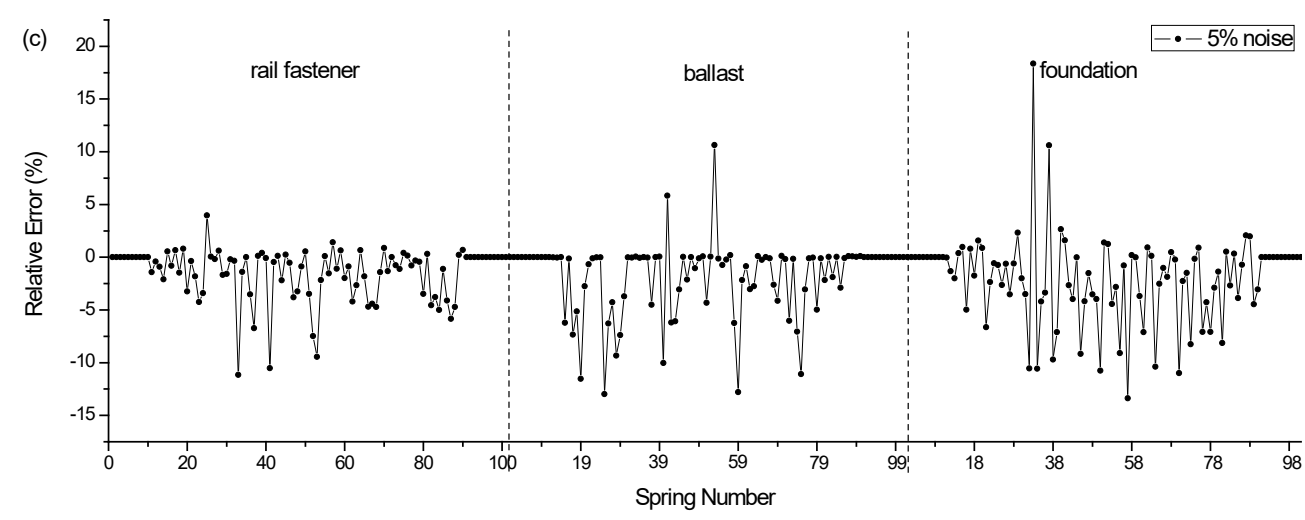

(b) Identified results with $5 \%$ measurements noise

Figure 14: Relative error of the whole substructure

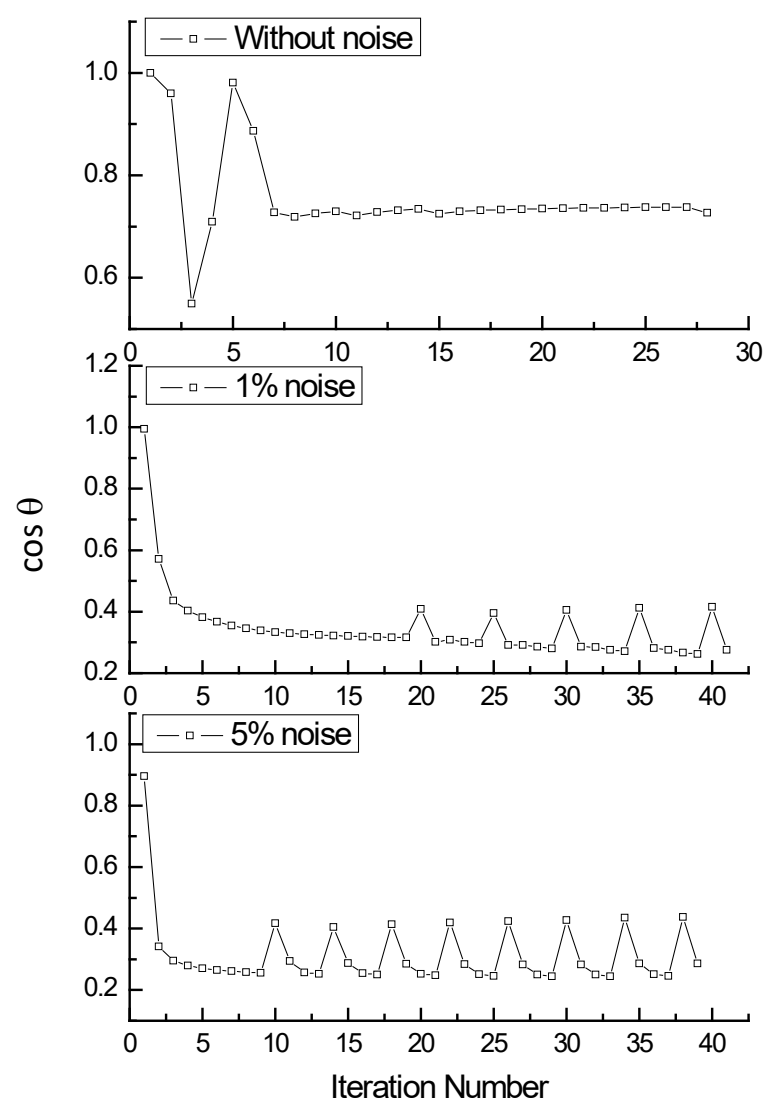

Figure 15: Evolution of $\cos \theta$ values with or without the measurement noise 


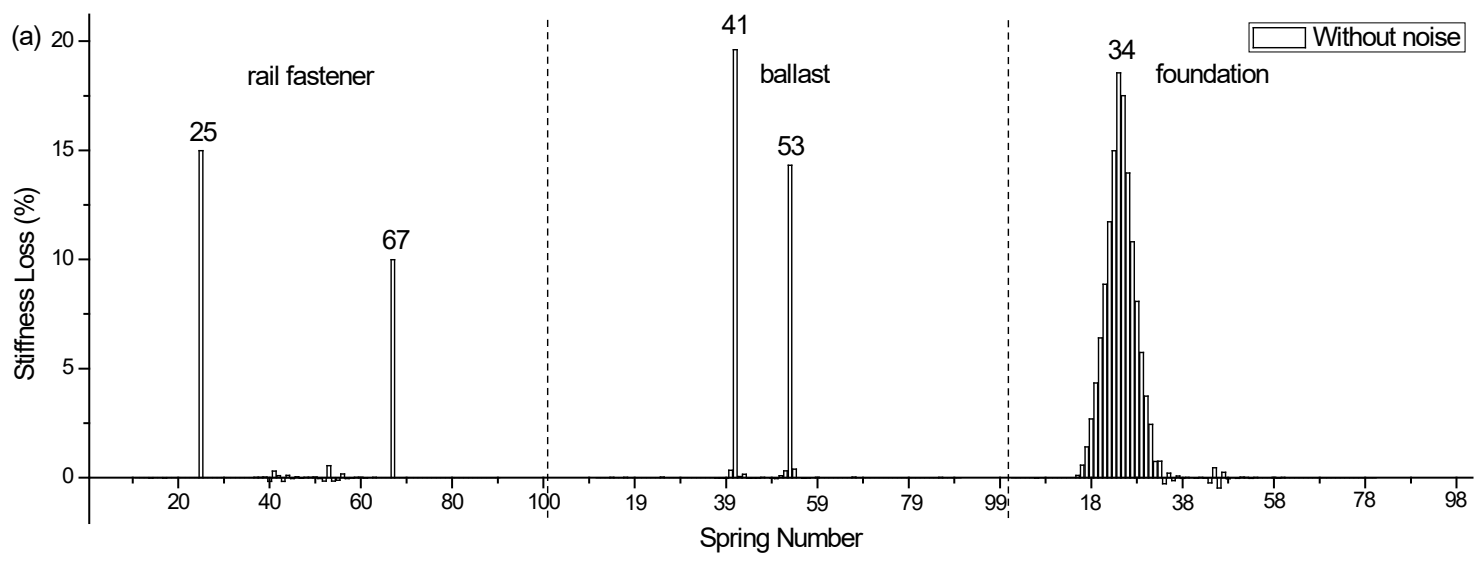

(a) Identified results without measurement noise

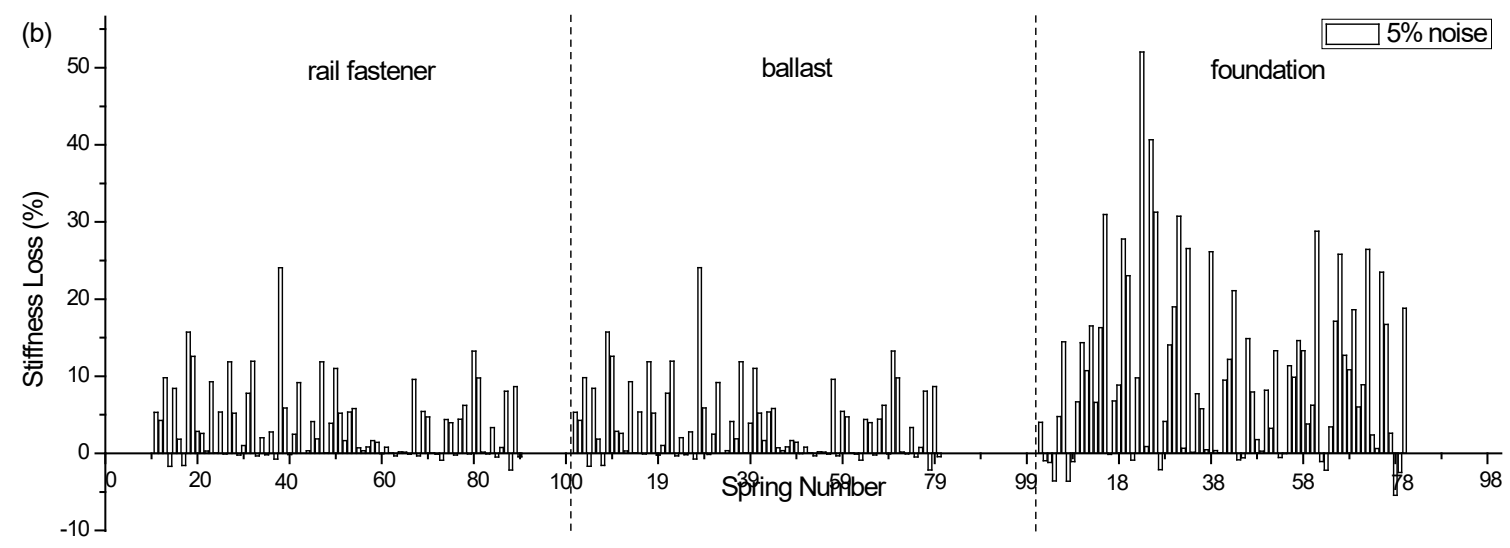

(b) Identified results with $5 \%$ measurement noise

Figure 16 Identification of track substructures with coupling components

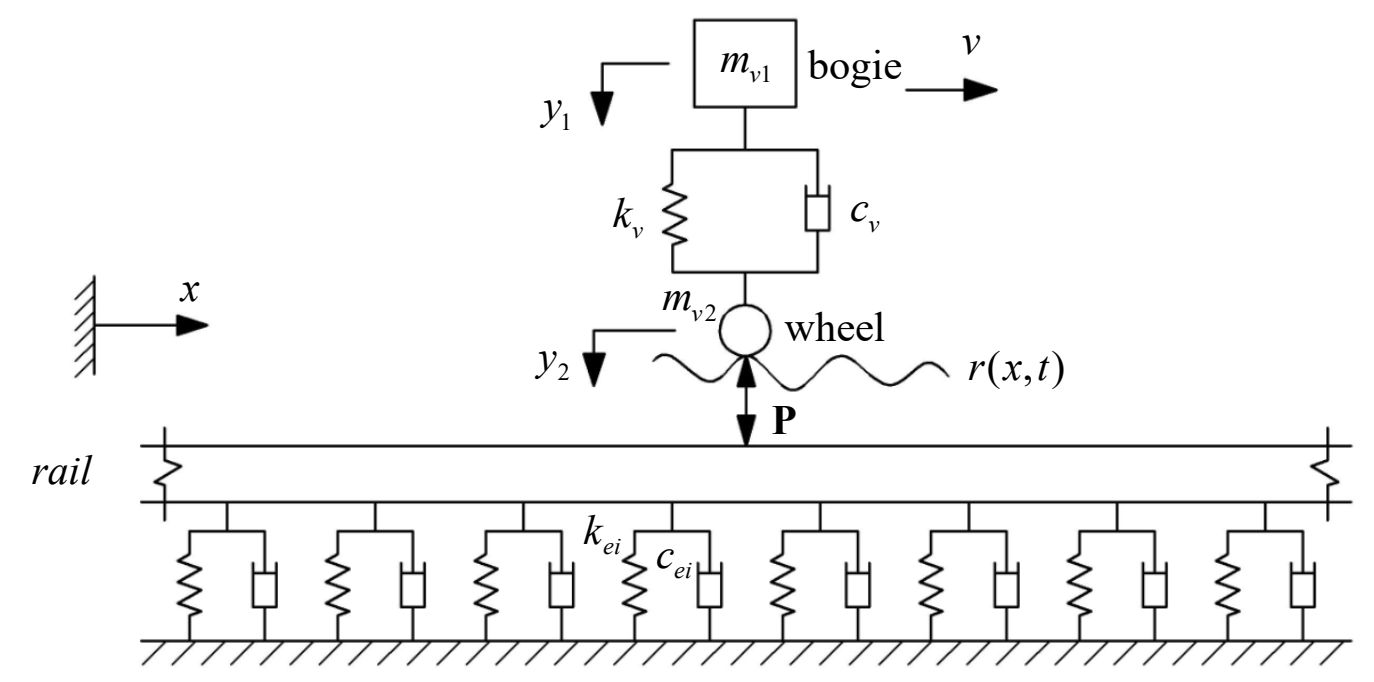

Figure 17 The simplified train vehicle model 


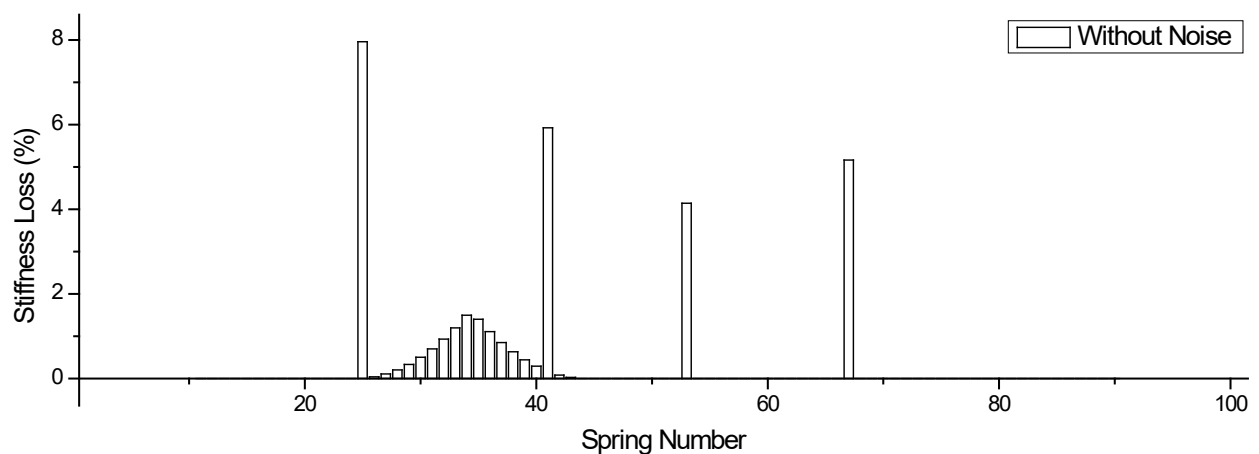

(a) Identification results without measurement noise

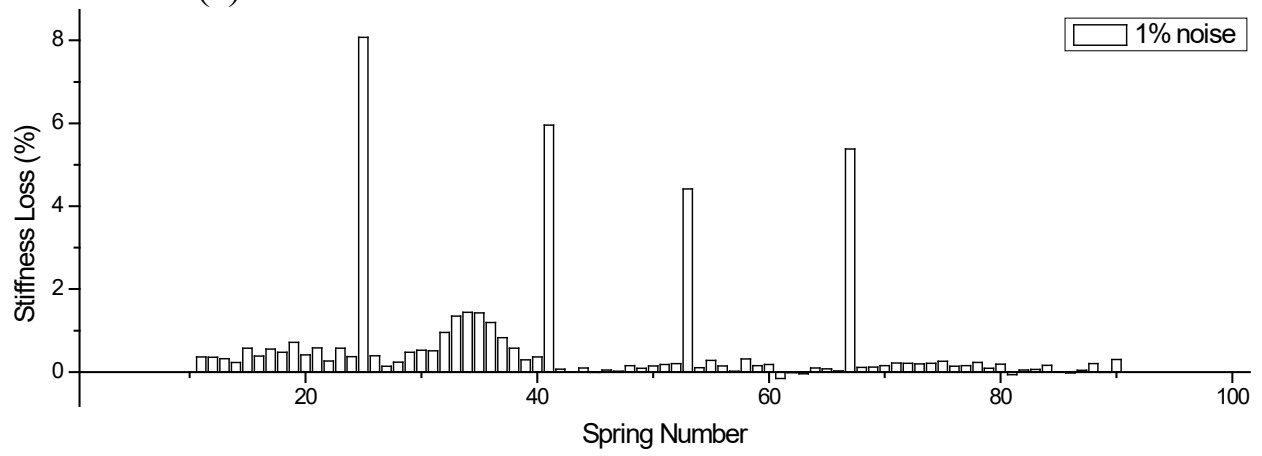

(b) Identification results with 1\% measurement noise

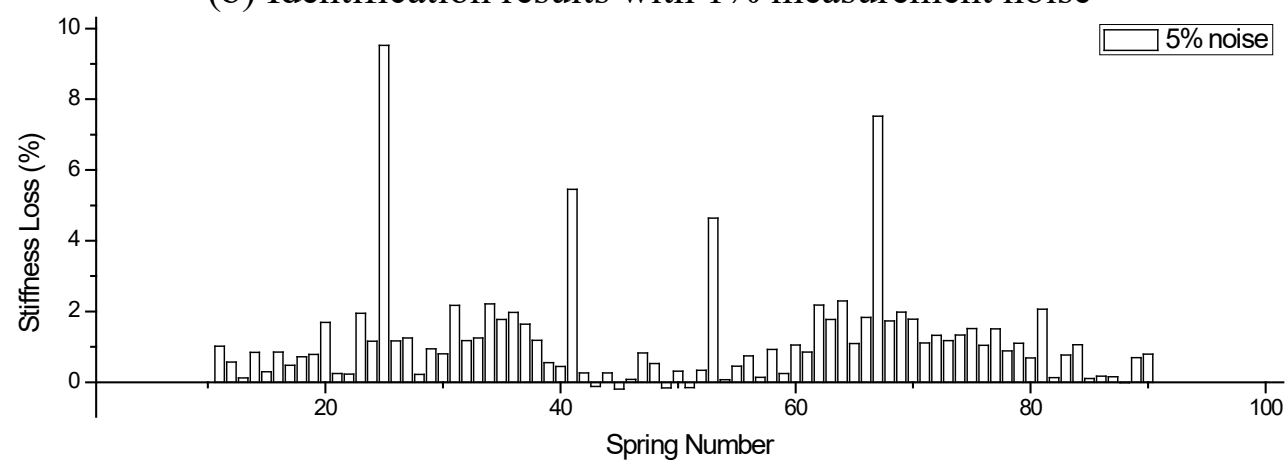

(c) Identification results with 5\% measurement noise Figure 18 Identified results with the equivalent track model 